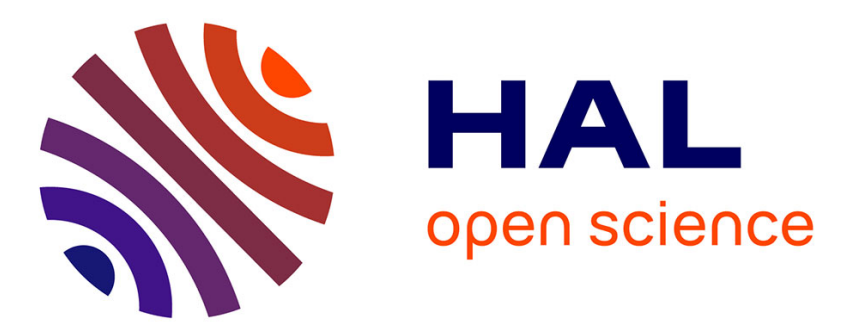

\title{
Non-linear dynamic thermomechanical behaviour of shape memory alloys
}

\author{
M.O. Moussa, Ziad Moumni, Olivier Doaré, Cyril Touzé, W. Zaki
}

\section{To cite this version:}

M.O. Moussa, Ziad Moumni, Olivier Doaré, Cyril Touzé, W. Zaki. Non-linear dynamic thermomechanical behaviour of shape memory alloys. Journal of Intelligent Material Systems and Structures, 2012, 23 (14), pp.1593-1611. 10.1177/1045389x12448446 . hal-00838865

HAL Id: hal-00838865

\section{https://hal-ensta-paris.archives-ouvertes.fr/hal-00838865}

Submitted on 12 Mar 2016

HAL is a multi-disciplinary open access archive for the deposit and dissemination of scientific research documents, whether they are published or not. The documents may come from teaching and research institutions in France or abroad, or from public or private research centers.
L'archive ouverte pluridisciplinaire HAL, est destinée au dépôt et à la diffusion de documents scientifiques de niveau recherche, publiés ou non, émanant des établissements d'enseignement et de recherche français ou étrangers, des laboratoires publics ou privés. 


\title{
Non-linear dynamic thermomechanical behaviour of shape memory alloys
}

\author{
Mohamed O Moussa, Ziad Moumni, Olivier Doaré, Cyril Touzé \\ Unité de Mécanique (UME), ENSTA-ParisTech, Palaiseau Cedex, France
}

Wael Zaki

Khalifa University of Science, Technology and Research (KUSTAR), Abu Dhabi, UAE

\begin{abstract}
The non-linear dynamic thermomechanical behaviour of superelastic shape memory alloys is investigated. To this end, the Zaki-Moumni model, initially developed for quasi-static loading cases, is extended to simulate the uniaxial forced oscillations of a shape memory alloy device. First, the influence of loading rate is accounted for by considering the thermomechanical coupling in the behaviour of $\mathrm{NiTi}$ shape memory alloy. Comparisons between simulations and experimental results show good agreement. Then, the forced response of a shape memory alloy device is investigated at resonance. Both isothermal and non-isothermal conditions are studied, as well as non-symmetric tensile-compressive restoring force. In the case of large values of forcing amplitudes, simulation results show that the dynamic response is prone to jumps, bifurcations and chaotic solutions.
\end{abstract}

\section{Keywords}

dynamic behaviour, hysteresis, shape memory alloy, pseudoelasticity, chaos, symmetry breaking, vibrations, thermomechanical coupling, natural frequencies

\section{Introduction}

The unusual behaviour of shape memory alloys (SMAs) is due to their ability to undergo martensitic transformation (Funakubo, 1987; Patoor and Berveiller, 1990). Beyond a certain temperature $A_{f}$, mechanical loading may lead to high inelastic deformation of the material that can be recovered by unloading. This so-called pseudoelastic behaviour is usually accompanied by considerable dissipation of energy and by stiffness variation, which can be used advantageously for vibration damping according to Saadat et al. (2002). Strain rate effects on the pseudoelastic characteristics have been the focus of a number of published articles from theoretical and experimental points of view. It is well established that increasing the strain rate leads to an increase in the slopes of the plateaus of forward and reverse martensitic transformations and leads to a change in the area of the hysteresis loop, which measures the dissipated energy.

Humbeeck and Delaey (1981) appear to be the first to underline the exothermic/endothermic characteristic of forward/reverse transformations and its influence on the hysteresis loop.
Experiments carried out at different strain rates and initial temperatures show an increase in stress thresholds with respect to initial temperature and strain rate in Mukherjee et al. (1985). A relationship between phase transformation stress and temperature was given by Leo et al. (1993). It allowed establishing a thermomechanical diagram by Leo et al. (1993). The dependency of stress thresholds was also highlighted in the experimental work by Shaw and Kyriakides (1995). However, according to the study done by Lin et al. (1996), variations in both dissipated energy and phase change thresholds occur only above a certain strain rate. From those studies emerges the question of distinguishing the most prominent effect between temperature and strain rate. On the one hand, Nemat-Nasser and Wei-Guo (2006) showed that pseudoelastic behaviour of NiTi SMAs is influenced by temperature variation induced by increasing the strain rate. On the other hand, Helm and Haupt (2002) argue that the dependency of pseudoelastic behaviour on strain rate is due to the viscosity of the SMAs. 
This divergence on the origin of strain rate effects remains in literature until recent works in this area. In particular, the experimental results shown by Grabe in Grabe and Bruhns (2008), obtained by finely controlling material temperature at different strain rates, allow to discard the assumption of the viscosity of SMAs. Morin et al. (2011) developed a model that accounts for the increase of the slope of transformation plateaus and the change in the dissipated energy with respect to strain rate by taking into account the influence of thermomechanical coupling. In addition, Chrysochoos et al. (1996) presented a thermomechanical model, after investigating temperature measurement using infrared thermography, which considers only the generated latent heat as a heating source. Since we are interested in the dynamic behaviour of SMAs, thermomechanical coupling must be considered when investigating dynamic response of a SMA device.

Regarding inertia effect and dynamics of SMAs structures, Machado et al. (2003) and Savi and Pacheco (2002) studied the dynamic behaviour of oneand two-degree-of-freedom SMA oscillators. A constitutive model is derived considering a non-convex polynomial free energy potential for the SMA, which depends on total strain and temperature as the only state variables. For lower temperatures, the polynomial possesses two minima representing stable martensite states, whereas for higher temperatures, a unique minimum exists corresponding to stable austenite. The explicit dependence on temperature allows the model to capture the salient features of the pseudoelastic and shape memory responses of the SMA in a simple and straightforward way. However, the model does not account for intrinsic mechanical dissipation, which contributes to the overall damping capacity of the system. The same SMA constitutive model is used by Savi et al. (2002) in order to analyse free and forced vibrations of a two-bar Von Mises frame structure. Constitutive and structural non-linearities are shown to have significant influence on the dynamic response of the frame, which is found to be highly non-linear with variable number of equilibrium states depending on temperature. Lagoudas et al. (2005) investigated a SMA-based passive damping system consisting of a mass attached to SMA wires. The experiments were carried out at a higher temperature where the material response was pseudoelastic. The authors measured vibration transmissibility and found it to decrease with increasing excitation frequency. The resonant frequency of the system was also found to decrease because of the softening behaviour due to the apparent stiffness of the SMA during phase transformation. Numerical simulations were performed using a constitutive model by Qidwai and Lagoudas (2000) specialized to the uniaxial SMA response, where temperature variations measured in the SMA wires are used as input. Strong thermomechanical coupling was considered in a subsequent article by Machado et al. (2009). Similar analysis was undertaken by Sitnikova et al. (2010) who considered an impact oscillator system consisting of an oscillator and a separate SMA support. When the vibration amplitude of the oscillator exceeds a certain threshold, impact against the support leads to energy dissipation by means of phase transformation in the SMA. Savi et al. (2008) used a uniaxial version of a constitutive model in order to simulate the dynamic response of a simple SMA oscillator. The model features four dissipative state variables that account for phase transformations between austenite, twinned martensite and martensite variants detwinned in tension and in compression. The use of different martensite volume fractions for the tensile and compressive martensite variants allows the model to account for tensilecompressive asymmetry. The equations of motion are solved using a Runge-Kutta iterative scheme, and the dissipative variables are updated using closest-point projection to enforce consistency with the phase transformation functions. Bernardini and co-workers investigated thoroughly the non-linear dynamic responses of a SMA device in a series of articles (Bernardini, 2001; Bernardini and Pence, 2005; Bernardini and Rega, 2009; Bernardini and Vestroni, 2003; Lacarbonara et al., 2004). Based on the Ivshin-Pence thermomechanical model and using robust path-following continuation methods, they draw out a complete picture of the resonant behaviour of the SMA device from small to medium amplitude range. The isothermal and nonisothermal conditions were investigated, and numerous non-linear phenomena (bifurcations, jumps, chaotic motions) and the complete picture of the influence of the model parameters were reported. Other works by Seelecke (2002) investigate free and forced vibrations of a SMA torsional pendulum, while chaotic responses of two coupled SMA oscillators were reported in Machado et al. (2003). Lagoudas et al. also investigated the thermomechanical dynamic behaviour of a SMA device in Hartl et al. (2010).

All these investigations and issues show that the non-linear frequency-response functions (FRFs) of SMAs exhibit a softening behaviour, as a consequence of the decrease of effective stiffness at the starting point of martensitic transformation. Jump phenomenon was also predicted. On the experimental side, softening effect was independently reported in many publications (e.g. Collet et al., 2001; Feng and Li, 1996; Schmidt and Lammering, 2004). However, jump phenomenon was almost not observed, being hidden by the increase of damping capacity. Recently, measurements on a torsional pendulum clearly evidenced the jump (Doare et al., 2011; Sbarra et al., 2011).

The main objective of the current contribution is to extend the model developed by Zaki (2010) and Zaki and Moumni (2007a) in quasi-static conditions, in order to study the general dynamic behaviours of structures 
made of SMAs. As already underlined, thermomechanical coupling is a key issue for correctly reproducing experimental observations. Section 'Model equations' is devoted to an introduction of the thermomechanical coupling in the Zaki-Moumni (ZM) model. Simulation of a tensile test on a cylinder shows that the main effects are well reproduced.

In order to validate the model in dynamic situations, as a necessary step before using it for full threedimensional (3D) simulations of structures made of SMAs, the 3D model (ZM) is reduced to a single degree of freedom, allowing for exhaustive comparisons with the numerical results given in the articles by Bernardini (2001), Bernardini and Pence (2005), Bernardini and Rega (2009), Bernardini and Vestroni (2003) and Lacarbonara et al. (2004). One main difference with their work lies in the fact that the thermodynamic admissibility is ensured in the ZM model thanks to the framework of generalized standard materials (GSMs) with internal constraints, developed by Moumni et al. (2008), used to derive the equations of the model. The GSM framework ensures the verification of the second law of thermodynamics.

Section 'Reduced model for a uniaxial SMA oscillator' explains the model reduction to a SMA device. Numerical results are then compared to those given by Lacarbonara et al. (2004), showing good agreement. New results, such as asymmetric restoring force in tensilecompression loadings, are also studied. All these results show the useful properties of the non-linear dynamic behaviour of SMAs in vibration control for instance.

\section{Model equations}

\section{ZM 3D thermomechanical model for SMAs}

Equations in isothermal conditions. The ZM model for SMAs is cast within the framework of GSMs with internal constraints, presented in Moumni et al. (2008). It was first introduced by Moumni (1995) for pseudoelastic behaviour and later generalized to take into account, in a unified way, all features associated with SMA (shape memory effect and reorientation) by Zaki and Moumni (2007b), cyclic SMA behaviour and training by Zaki and Moumni (2007a), tension-compression asymmetry by Zaki (2010) and irrecoverable plastic deformation of martensite by Zaki et al. (2010). The original ZM model uses the following expression of the Helmholtz free energy for the derivation of constitutive relations

$$
\begin{aligned}
\mathcal{W}_{\text {iso }}= & (1-z)\left[\frac{1}{2} \varepsilon_{\mathrm{A}}: \boldsymbol{S}_{\mathrm{A}}^{-1}: \varepsilon_{\mathrm{A}}\right] \\
& +z\left[\frac{1}{2}\left(\varepsilon_{\mathrm{M}}-\varepsilon_{\text {ori }}\right): \boldsymbol{S}_{\mathrm{M}}^{-1}:\left(\varepsilon_{M}-\varepsilon_{o r i}\right)+C(T)\right] \\
& +G \frac{z^{2}}{2}+\frac{z}{2}[\alpha z+\beta(1-z)]\left(\frac{2}{3} \varepsilon_{o r i}: \varepsilon_{o r i}\right)
\end{aligned}
$$

In the above equation, $\varepsilon_{A}$ and $\varepsilon_{M}$ are the local strain tensors of austenite and martensite, respectively; $T$ is the temperature; $z$ is the volume fraction of martensite and $\varepsilon_{\text {ori }}$ is the orientation strain tensor (note that the tensors and vectors are written with bold font in equations). $\boldsymbol{S}_{A}$ and $\boldsymbol{S}_{M}$ are the compliance tensors of austenite and martensite. $G, \alpha$ and $\beta$ are material parameters that influence the shape of the superelastic hysteresis loop and the slopes of the stress-strain curve during phase change and martensite orientation. $C(T)$ is an energy density that depends on temperature as follows

$$
C(T)=\xi\left(T-T^{0}\right)+\kappa
$$

where $\xi$ and $\kappa$ are material parameters associated with the density of the generated latent heat.

The state variables obey the following physical constraints:

- The macroscopic strain tensor $\varepsilon$ is an average over the representative elementary volume (REV) of the strain within the austenite and martensite phases. By construction, $\varepsilon$ is given by

$$
(1-z) \boldsymbol{\varepsilon}_{A}+z \boldsymbol{\varepsilon}_{M}-\boldsymbol{\varepsilon}=0
$$

- The equivalent orientation strain cannot exceed a maximum detwinning strain $\varepsilon_{\max }$

$$
\boldsymbol{\varepsilon}_{\max }-\sqrt{\frac{2}{3} \boldsymbol{\varepsilon}_{\text {ori }}: \boldsymbol{\varepsilon}_{\text {ori }}} \geq 0
$$

The above constraints derive from the following potential

$$
\begin{aligned}
W_{1}= & -\lambda:\left[(1-z) \boldsymbol{\varepsilon}_{A}+z \boldsymbol{\varepsilon}_{M}-\boldsymbol{\varepsilon}\right] \\
& -\mu\left(\boldsymbol{\varepsilon}_{\text {max }}-\sqrt{\frac{2}{3} \boldsymbol{\varepsilon}_{\text {ori }}: \boldsymbol{\varepsilon}_{\text {ori }}}\right)-\nu_{1} z-\nu_{2}(1-z)
\end{aligned}
$$

where the Lagrange multipliers $\nu_{1}, \nu_{2}$ and $\mu$ are such that

$$
\begin{gathered}
\nu_{1} \geq 0, \nu_{1} z=0 \\
\nu_{2} \geq 0, \nu_{2}(1-z)=0
\end{gathered}
$$

and

$$
\mu \geq 0, \mu\left(\varepsilon_{\max }-\sqrt{\frac{2}{3} \varepsilon_{\text {ori }}: \boldsymbol{\varepsilon}_{\text {ori }}}\right)=0
$$

The sum of the Helmholtz energy density (equation (1)) and the potential $W_{1}$ (equation (5)) gives the Lagrangian $\mathcal{L}$, which is then used to derive the state equations. With some algebra, the following stressstrain relation is obtained

$$
\boldsymbol{\sigma}=\boldsymbol{S}^{-1}:\left(\boldsymbol{\varepsilon}-z \boldsymbol{\varepsilon}_{\text {ori }}\right)
$$


where $S$ is the equivalent compliance tensor of the material, given by

$$
\boldsymbol{S}=(1-z) \boldsymbol{S}_{A}+{ }_{z} \boldsymbol{S}_{M}
$$

One can also derive the state equations for two thermodynamical forces $\mathcal{A}_{z}$ and $\mathcal{A}_{\text {ori }}$ associated with $z$ and $\boldsymbol{\varepsilon}_{\text {ori }}$, respectively, and given by

$$
\begin{aligned}
\mathcal{A}_{z}= & \frac{-\partial \mathcal{L}}{\partial z} \\
= & \frac{-1}{2} S_{A}^{-1}: \sigma: \sigma+\frac{1}{2} S_{M}^{-1}: \sigma: \sigma+\sigma: \varepsilon_{o r i} \\
& -C(T)-G z-\left((\alpha-\beta) z+\frac{\beta}{2}\right)+\left(\frac{2}{3} \varepsilon_{o r i}: \varepsilon_{o r i}\right)
\end{aligned}
$$

and

$$
\begin{aligned}
\mathcal{A}_{\text {ori }} & =\frac{-\partial \mathcal{L}}{\partial \varepsilon_{\text {ori }}} \\
& =z \sigma-z(\alpha z+(1-z) \beta) \varepsilon_{\text {ori }}-\frac{2}{3} \mu \frac{\varepsilon_{\text {ori }}}{\sqrt{\frac{2}{3} \varepsilon_{\text {ori }}: \varepsilon_{\text {ori }}}}
\end{aligned}
$$

Then, the thermodynamic forces are taken as subgradients of a pseudopotential of dissipation $D$ defined by

$$
D=[a(1-z)+b z]|\dot{z}|+z^{2} Y \sqrt{\frac{2}{3} \dot{\boldsymbol{\varepsilon}}_{\text {ori }}: \dot{\boldsymbol{\varepsilon}}_{\text {ori }}}
$$

where $a$ and $b$ are positive material parameters and $Y$ is the orientation yield stress in tension. This allows definition of yield functions for phase change $\left(\mathcal{F}_{z}^{1}\right.$ and $\left.\mathcal{F}_{z}^{2}\right)$ and for martensite orientation $\left(\mathcal{F}_{o r i}\right)$. The evolution of the state variables $z$ and $\boldsymbol{\varepsilon}_{\text {ori }}$ is governed by the consistency conditions associated with the yield functions. If the orientation-finish stress is lower than the critical stress for forward phase change (i.e. if stress finish $\sigma_{r f}$ of the detwinning process is lower than the stress start $\sigma_{m s}$ of the phase change), the model is such that the stress-induced martensite is completely oriented as soon as forward phase change begins.

Thermomechanical coupling. It is worth mentioning that in the classical model previously presented, the Helmholtz free energy does not account for the heat capacity of SMA. The current section aims at including a constant heat capacity $C_{P}$, assuming that it is the same in austenite and martensite phases. Therefore, the augmented expression of the Helmholtz free energy reads

$$
\mathcal{W}=\mathcal{W}_{\text {iso }}+\rho C_{\mathrm{P}}\left(T-T^{0}-T \ln \left(\frac{T}{T^{0}}\right)\right)
$$

where $\rho$ is the mass density. Besides, considering a constant heat capacity involves changes only in the state equation of the entropy. The latter now reads

$$
\eta=-\frac{\partial \mathcal{W}}{\partial T}=-\xi z+\rho C_{P} \ln \left(\frac{T}{T^{0}}\right)
$$

Then, the first law of thermodynamics can be written in terms of the Helmholtz free energy as

$$
\dot{\mathcal{W}}+T \dot{\eta}+\eta \dot{T}=\boldsymbol{\sigma}: \dot{\varepsilon}-\operatorname{div}(\boldsymbol{q})+S_{v}
$$

where $q$ is the heat influx and $S_{v}$ is the density of the internal heat generation.

The second law of thermodynamics gives

$$
\mathcal{D}=T \dot{\eta}+\operatorname{div}(\boldsymbol{q})-\frac{\boldsymbol{q} \nabla T}{T}-S_{v} \geq 0
$$

Using the first and the second laws simultaneously, one deduces that

$$
\mathcal{D}=\boldsymbol{\sigma}: \dot{\varepsilon}-(\dot{\mathcal{W}}+\dot{T} \eta)-\frac{\boldsymbol{q} \nabla T}{T} \geq 0
$$

where $\mathcal{D}_{\text {th }}=-\boldsymbol{q} \nabla T / T$ is the heat dissipation.

Using the differentiated expression of $\mathcal{W}$ and $\eta$

$$
\dot{\mathcal{W}}=\frac{\partial \mathcal{W}}{\partial \varepsilon}: \dot{\varepsilon}+\frac{\partial \mathcal{W}}{\partial z} \dot{z}+\frac{\partial \mathcal{W}}{\partial \varepsilon_{\text {ori }}}: \dot{\varepsilon}_{\text {ori }}+\frac{\partial \mathcal{W}}{\partial z} \dot{z}+\frac{\partial \mathcal{W}}{\partial T} \dot{T}
$$

and

$$
\dot{\eta}=\frac{\partial \eta}{\partial \varepsilon}: \dot{\varepsilon}+\frac{\partial \eta}{\partial z} \dot{z}+\frac{\partial \eta}{\partial \varepsilon_{o r i}}: \dot{\varepsilon}_{o r i}+\frac{\partial \eta}{\partial z} \dot{z}+\frac{\partial \mathcal{W}}{\partial T} \dot{T}
$$

the dissipation can be given, thanks to $\eta=-\partial \mathcal{W} / \partial T$, $\mathcal{A}_{z}=-\partial \mathcal{W} / \partial z$ and $\mathcal{A}_{\text {ori }}=\partial \mathcal{W} / \partial \varepsilon_{\text {ori }}$, in terms of the Helmholtz free energy as

$$
\begin{aligned}
\mathcal{D}= & \mathcal{D}_{\text {int }}-T \frac{\partial^{2} \mathcal{W}}{\partial T \partial \varepsilon}: \dot{\varepsilon}-T \frac{\partial^{2} \mathcal{W}}{\partial T \partial \varepsilon_{o r i}}: \dot{\varepsilon}_{\text {ori }} \\
& -T \frac{\partial^{2} \mathcal{W}}{\partial T \partial z} \dot{z}-T \frac{\partial^{2} \mathcal{W}}{\partial T^{2}} \dot{T}+\operatorname{div}(\boldsymbol{q})-\frac{\boldsymbol{q} \nabla T}{T}-S_{v}
\end{aligned}
$$

The second right-hand term accounts for thermoelastic effects, the third for the dissipation generated by the orientation of martensite, the fourth for the latent heat and the fifth for the heat capacity.

Assuming that the intrinsic dissipation $\mathcal{D}_{\text {int }}$ is positive, one can write

$$
\mathcal{D}_{\text {int }}=\mathcal{A}_{z} \dot{z}+\mathcal{A}_{\text {ori }}: \dot{\varepsilon}_{\text {ori }} \geq 0
$$

Moreover, using Fourier's law of heat conduction, $\boldsymbol{q}=-K_{v} \nabla T$, where $K_{v}$ is the thermal conductivity assumed to be the same in both phases, allows one to 
derive the heat equation after neglecting the thermoelastic effects

$\rho C_{P} \dot{T}-\operatorname{div}\left(K_{v} \nabla T\right)=-T \frac{\partial \mathcal{A}_{\text {ori }}}{\partial T}: \dot{\boldsymbol{\varepsilon}}_{\text {ori }}-T \frac{\partial \mathcal{A}_{z}}{\partial T} \dot{z}+S_{v}+\mathcal{D}_{\text {int }}$

Besides, complete martensite orientation is assumed at the beginning of forward phase change, leading to the following expression of the martensite orientation strain, in the case of proportional loading

$$
\varepsilon_{\text {ori }}=\varepsilon_{\max }\left(\frac{\boldsymbol{\sigma}^{\prime}}{\boldsymbol{\sigma}_{V M}}\right)
$$

where $\varepsilon_{\max }$ is the maximum orientation strain, $\boldsymbol{\sigma}^{\prime}$ the deviatoric stress tensor and $\sigma_{V M}$ the Von Mises equivalent stress.

Indeed, while only the phase change phenomenon involves dissipation, heat generation due to martensite orientation is null and heat equation reads now

$$
\rho C_{P} \dot{T}-\operatorname{div}\left(K_{v} \nabla T\right)=-T \frac{\partial \mathcal{A}_{z}}{\partial T} \dot{z}+\mathcal{A}_{z} \dot{z}
$$

Natural convection is assumed at the boundary $(\partial \Omega)$ such that

$$
Q_{\partial \Omega}=h\left(T_{e x}-T\right)
$$

where $T_{e x}$ is the temperature of the surrounding medium and $h$ is the heat convection coefficient.

Thermomechanical coupling leads to the increase of the slope of the transformation plateaus in stress-strain space with respect to strain rate: the completion of the phase transformation becomes more difficult at high strain rate (Figure 1), and the material temperature increases, which influences its mechanical response. It is well known, in addition, that the area of the

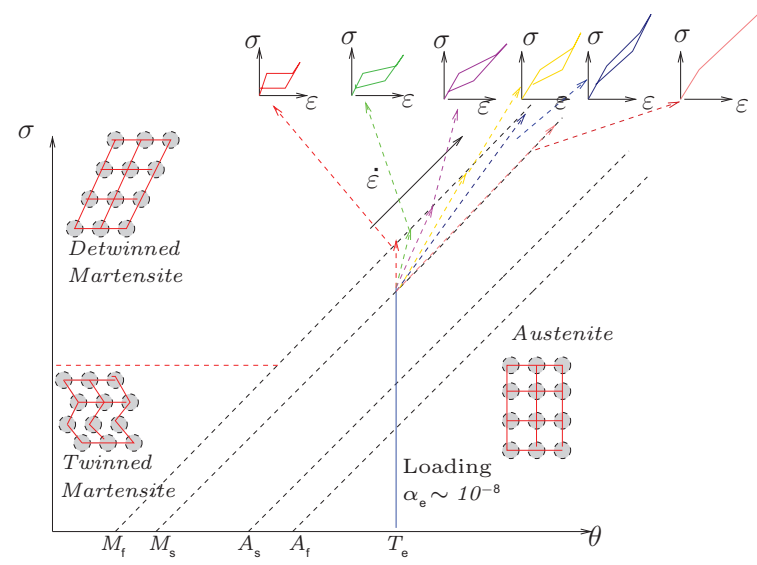

Figure I. Stress-temperature diagram showing that any change in the temperature for different strain rates involves different mechanical behaviours.

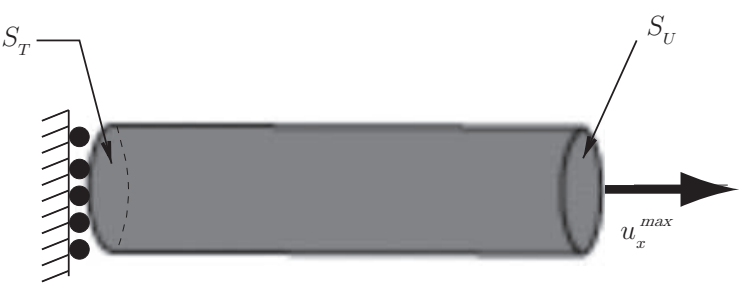

Figure 2. Meshed structure $\Omega$ with boundary zones.

hysteretic loop changes while increasing strain rate (Grabe and Bruhns, 2008; Hartl et al., 2010; He and Sun, 2011; Lexcellent and Rejzner, 2000; Morin et al., 2011).

In order to verify the ability of the model to recover properly, these two effects, a finite element (FE) model of a wire of length $10^{-1} \mathrm{~m}$ and a circular cross section of radius $10^{-3} \mathrm{~m} \mathrm{~m}$, have been implemented with a behaviour law dictated by ZM model. The discretization is performed, thanks to 500 cubic elements with 20 nodes (Gauss's points) where mechanical fields are evaluated. There are three degrees of freedom by node and, therefore, 30,000 degrees of freedom in total.

Thus, at any node N1 of the meshing $\Omega$ (Figure 2), one solves the following set of equations:

Mechanical equilibrium and heat equations

$$
\operatorname{div}(\boldsymbol{\sigma})=0, \quad \forall \mathrm{N} 1 \in \Omega
$$

$\rho C_{P} \dot{T}-\operatorname{div}\left(K_{v} \nabla T\right)=-T \frac{\partial \mathcal{A}_{z}}{\partial T} \dot{z}+\mathcal{A}_{z} \dot{z}, \quad \forall \mathrm{N} 1 \in \Omega$

ZM model

$$
\boldsymbol{\sigma}=\boldsymbol{S}^{-1}:\left(\boldsymbol{\varepsilon}-z \boldsymbol{\varepsilon}_{\text {ori }}\right), \quad \forall \mathrm{N} 1 \in \Omega
$$

$$
\mathcal{A}_{z} \in \partial_{\dot{z}} \mathcal{D}, \quad \forall \mathrm{N} 1 \in \Omega
$$

Mechanical boundary conditions

$$
\begin{gathered}
u_{x}=0, \quad \forall \mathrm{N} 1 \in S_{T} \\
u_{x}^{\max }=16 \times 10^{-3} \mathrm{~m} \quad \forall \mathrm{N} 1 \in S_{U}
\end{gathered}
$$

Thermal boundary conditions:

$$
q=Q_{\partial \Omega}=h\left(T_{e x}-T\right), \quad \mathrm{N} 1 \in \partial \Omega
$$

where $S_{T}$ and $S_{U}$ are the boundary areas at $x=0$ and $x=0.15$, respectively (see Figure 2). $\partial \Omega$ stands for the whole boundary area, that is, including $S_{T}, S_{U}$ and the lateral surface. Fifty loading increments and physical three time steps $(d t=40,0.4$ and $0.004 \mathrm{~s})$ are used in 
Table I. ZM parameters corresponding to the identified parameters in Morin et al. (201I).

\begin{tabular}{ll}
\hline Symbol & Value \\
\hline$E_{A}$ & $615 \times 10^{8} \mathrm{~Pa}$ \\
$E_{M}$ & $2.4 \times 10^{9} \mathrm{~Pa}$ \\
$\mu$ & 0.3 \\
$\alpha$ & $275 \times 10^{7} \mathrm{~Pa}$ \\
$Y$ & $11 \times 10^{7} \mathrm{~Pa}$ \\
$\xi$ & $0.2914 \times 10^{6} \mathrm{~Pa} /{ }^{\circ} \mathrm{C}$ \\
$\varepsilon_{\max }$ & $4.0 \%$ \\
$C_{p}$ & $440 \mathrm{~kg}^{-1} \mathrm{~K}^{-1}$ \\
$\rho$ & $6500 \mathrm{~kg} \mathrm{~m}^{-3}$ \\
$b$ & $6.9091 \times 10^{6} \mathrm{~Pa}$ \\
$G$ & $4.6556 \times 10^{6} \mathrm{~Pa}$ \\
$\beta$ & $55 \times 10^{8} \mathrm{~Pa}$ \\
$A_{f}$ & $40^{\circ} \mathrm{C}$ \\
$\kappa$ & $6.8920 \times 10^{6} \mathrm{~Pa}$ \\
$k_{v}$ & $18 \mathrm{~W} \mathrm{~m} \mathrm{~K}^{-1}$ \\
$a$ & $6.8920 \times 10^{6} \mathrm{~Pa}$ \\
$h_{\text {water }}$ & $800 \mathrm{~W} \mathrm{~m} \mathrm{~K}^{-1}$ \\
$h_{\text {air }}$ & $50 \mathrm{~W} \mathrm{~m}^{-2} \mathrm{~K}^{-1}$ \\
\hline
\end{tabular}

ZM: Zaki-Moumni.

the FE simulations, conducted for increasing strain rates as follows: $4 \times 10^{-5} \mathrm{~s}^{-5}, \quad 4 \times 10^{-2} \mathrm{~s}^{-2}$ and $4 \times 10^{-1} \mathrm{~s}^{-1}$.

Table 1 gives the values of material parameters used for simulations as already identified in Morin et al. (2011). The selected node for showing simulation results is located far from the edges of the specimen in order to avoid boundary effects (according to the Saint-Venant principle). Using the above expression for $\varepsilon_{o r i}$ allows to avoid the computation of the consistency condition on the orientation yield function $\mathcal{F}_{\text {ori }}$.

The results of the simulation are shown in Figures 3 and 4. Figure 3(a) and (b) shows the simulated behaviour at a selected node within the post-processing zone for the three increasing values of the strain rate and for two heat exchange situations $(h=50$ and 800 $\mathrm{Wm}^{-2} \mathrm{~K}^{-1}$ ). The thermal hardening is well reproduced by the model for both the values of $h$. However, smaller the value of $h$, greater the effect of thermomechanical coupling: the slope variation of the martensitic transformation plateaus is increased about seven times between the responses at $\dot{\varepsilon}=4 \times 10^{-5} \mathrm{~s}^{-1}$ and $\dot{\varepsilon}=4 \times 10^{-2} s^{-1}$ for $h=50 \mathrm{Wm}^{-2} \mathrm{~K}^{-1}$ (Figure $3(\mathrm{~b})$ ). Physically, this effect is explained by the increase of the temperature with increasing strain rate, hence making the nucleation and growth of martensite more difficult. The size of the hysteresis loop is also shown to decrease in the FE simulation, which recovers the main observed features brought by the thermomechanical coupling with a fair qualitative accuracy.

Figure 4(a) and (b) shows the corresponding temperature variations during the simulated experiment, for two different strain rates: $4 \times 10^{-2} \mathrm{~s}^{-1}$ and $4 \times 10^{-5} \mathrm{~s}^{-1}$. The temperature variations are governed by the competition between mechanical dissipation and latent heat. One can observe that in zone I in Figure 4(a) and (b), the temperature remains constant because of the absence of thermoelastic coupling in the current numerical investigation. However, along zone II, the beginning of the exothermic phase transformation generates the temperature variations. Zone III corresponds to stabilization of the temperature between forward transformation finishing point and the reverse transformation starting one. The endothermic characteristic of the reverse transformation involves a decrease in the temperature evolution as evidenced in zone IV, where the reverse phase change begins. Finally, zone V shows no temperature evolution because of the

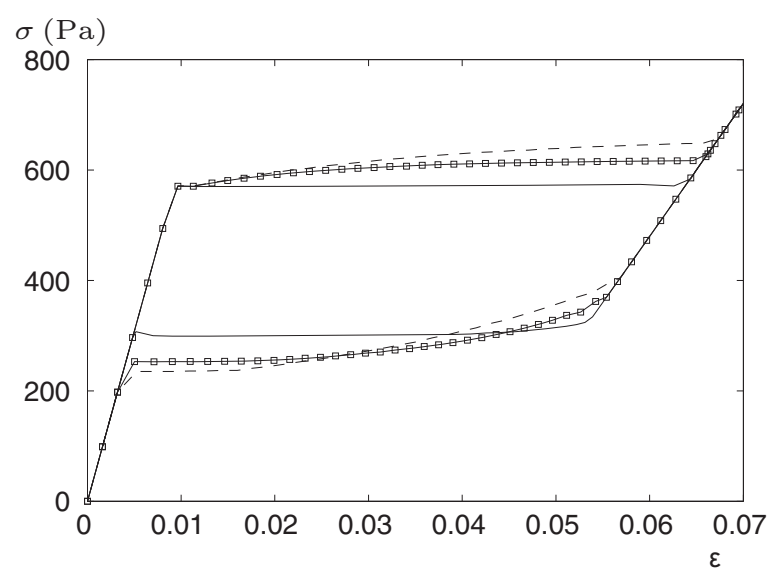

(a)

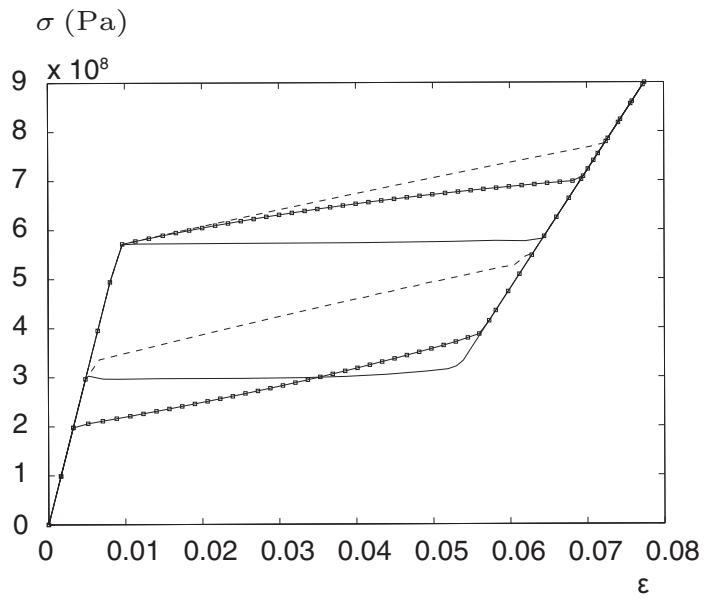

(b)

Figure 3. Influence of the strain rate on the stress-strain response: stress-strain curves at different strain rates $\dot{\varepsilon}=4 \times 10^{-5} \mathrm{~s}^{-1}$ (solid line), $\dot{\varepsilon}=4 \times 10^{-2} s^{-1}$ (square) and $\dot{\varepsilon}=4 \times 10^{-1} s^{-1}$ (dashed line): (a) for $h=800$ and (b) for $h=50$. 


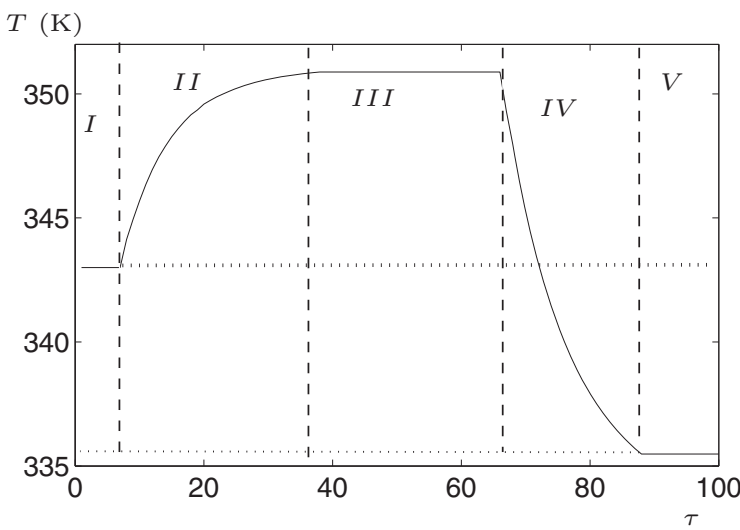

(a)

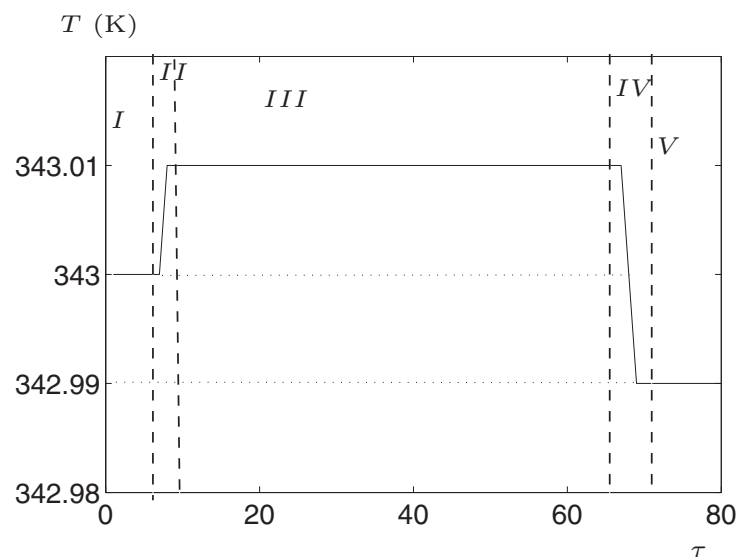

(b)

Figure 4. Temperature variation with time: (a) at $\dot{\varepsilon}=4 \times 10^{-2} \mathrm{~s}^{-1}$ and (b) at $\dot{\varepsilon}=\dot{\varepsilon}=4 \times 10^{-5} \mathrm{~s}^{-1}$.

vanishing of all heat sources in the current problem. However, one can observe that at the end, the temperature can reach a value below the initial temperature due to the competition between the latent heat and the heating exchange rate.

A quantitative comparison is now drawn out by using the data of the experiments shown in Shaw and Kyriakides (1995), at two different strain rates and where the external environment is air. Figure 5(a) and (b) shows the stress-strain responses at $4 \times 10^{-5} \mathrm{~s}^{-1}$ and $4 \times 10^{-2} \mathrm{~s}^{-1}$ for simulation and experiment. One can highlight that the material parameters used are the same for both figures; hence, only the strain rate effect is under consideration currently. The comparison, although with little mismatches, reveals the ability of our model to reproduce both the quasi-static behaviour and thermomechanical coupling effects.

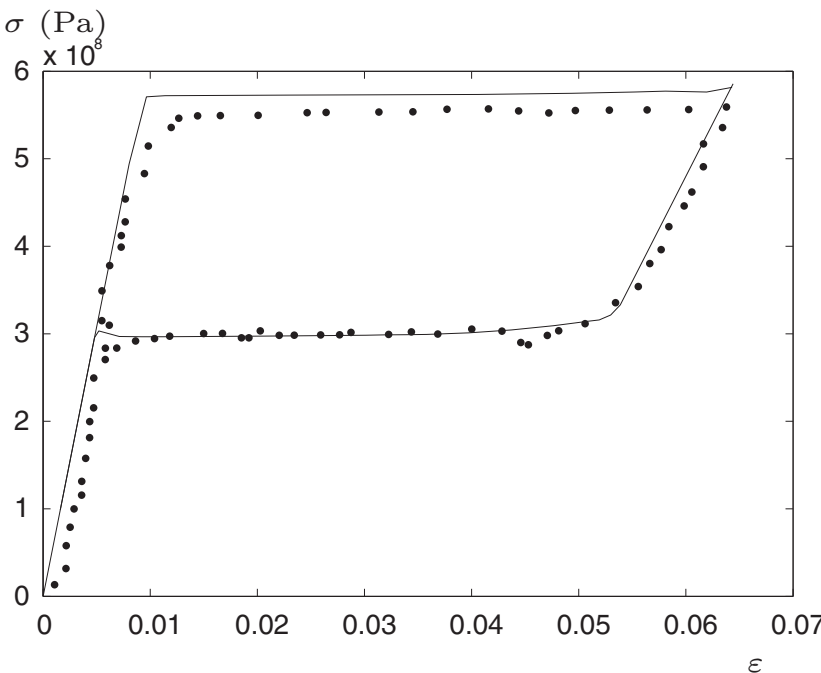

(a)
Since the 3D thermomechanical behaviour is validated on a simple quasi-static simulation, the remainder of the article is devoted to extending the model to handle the case of vibrations. For the purpose of validation, the $3 \mathrm{D}$ model will first be reduced to a single degree of freedom oscillator displaying pseudoelasticity (SMA device) in order to compare numerical results with the literature.

\section{Reduced model for a uniaxial SMA oscillator}

The 3D model presented above is now particularized to model a non-linear spring included in an oscillator. A schematic view of the system under consideration is given in Figure 6. More precisely, our goal is to derive

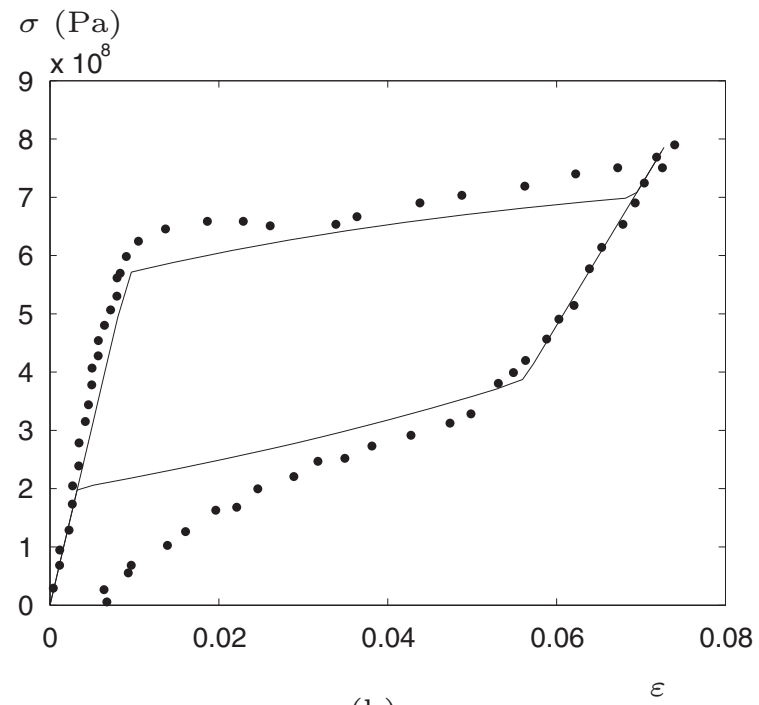

(b)

Figure 5. Stress-strain responses with ZM model (solid line) and Shaw and Kyriakides's experiment (dotted line) at (a) $\dot{\varepsilon}=4 \times 10^{-5} s^{-1}$ and (b) $\dot{\varepsilon}=4 \times 10^{-2} s^{-1}$.

ZM: Zaki-Moumni. 


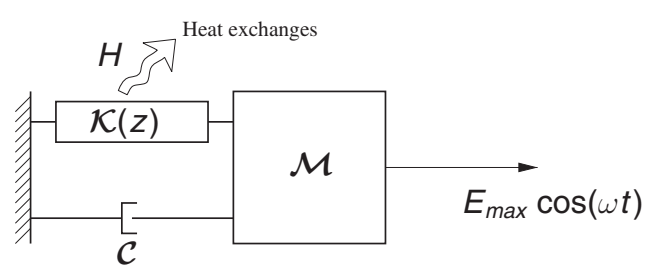

Figure 6. Schematic view of a SMA mass-spring oscillator with an external forcing.

SMA: shape memory alloy.

a non-linear spring law reproducing the SMA behaviour, so as to include it in a zero-dimensional oscillator equation. To that end, a $1 \mathrm{D}$ bar of cross-section $S$ and length $l$, clamped at $x=0$ and attached to the mass $\mathcal{M}$ at $x=l$, is considered, only for derivation of the non-linear spring behaviour law. One has to keep in mind that although the bar is $1 \mathrm{D}$, eventually the oscillator model is zero-dimensional. This remark will guide the retained choice for the spatial dependence of the temperature one has to account for when reducing the model. A damper $\mathcal{C}$ is mounted in parallel to model other sources of dissipation. An external force $F=F \mathbf{e}_{x}$ is exerted on $x=l$. Under the assumption that $l \gg D$, $D$ being a typical length characterizing the cross section, the stress in the SMA bar can be considered to be $1 \mathrm{D}, \sigma=\sigma \boldsymbol{e}_{x} \otimes e_{x}$, with

$$
\sigma=\frac{F}{S}
$$

One defines the total strain in the $x$-direction as

$$
\varepsilon=\frac{X}{l}
$$

where $X$ is the displacement of the system at $x=l$. Besides, the 1D detwinning strain reads

$$
\varepsilon_{o r i, x x}=\frac{X_{o r i}}{l}
$$

where $X_{\text {ori }}$ is an internal displacement due the martensitic detwinning process.

Equation (9) gives in 1D version

$$
\sigma=E_{e q}(z)\left(\varepsilon-z \varepsilon_{o r i}\right)
$$

where $E_{e q}(z)$ is the equivalent Young's modulus, given by

$$
E_{\text {eq }}=(1-z) E_{A}+z E_{M}
$$

Using equation (27), equation (28) and

$$
\varepsilon_{\text {ori }}=\frac{X_{\text {ori }}}{l}
$$

one can derive the relationship between the force exerted on the cylinder and its elongation as

$$
F=\mathcal{K}(z)\left(X-z X_{\text {ori }}\right)
$$

$\mathcal{K}(z)$ is a non-linear stiffness depending on the amount of martensitic phase $z$. Its expression in the single-degree-of-freedom case is analogous to equation (10) for the $3 \mathrm{D}$ case and reads

$$
\mathcal{K}(z)=\left(\frac{1-z}{\mathcal{K}_{a}}+\frac{z}{\mathcal{K}_{m}}\right)^{-1}
$$

where $\mathcal{K}_{a}=E_{a} S / l$ and $\mathcal{K}_{m}=E_{m} S / l$ are the stiffnesses of the austenitic and martensitic phases, respectively. In the context of 1D deformations, the thermodynamic force $\mathcal{A}_{z}$ now reads

$$
\begin{aligned}
\mathcal{A}_{z}= & \left(\frac{F}{S}\right)^{2}\left(\frac{1}{2 E_{m}}-\frac{1}{2 E_{a}}\right)+\frac{F X_{\text {ori }}}{S l}-C(T) \\
& -G z-\left[(\alpha-\beta) z+\frac{\beta}{2}\right]\left(\frac{X_{\text {ori }}}{l}\right)^{2}
\end{aligned}
$$

In this simplified approach, the detwinning criterion is disregarded, and the displacement $X_{\text {ori }}$ has the following form

$$
X_{o r i}=X_{\max } \operatorname{sgn}(F)
$$

where $\operatorname{sgn}(F)$ denotes the sign of $F$. This assumption implies that the detwinning process is fully achieved at any time during the transformation. Actually, the initial temperature is such that the detwinning finish force is smaller than the starting force of phase change: according to the phase diagram in Figure 1, the level of the detwinning process becomes maximum at such a temperature. Criteria functions have the following form in the $1 \mathrm{D}$ model

$$
\mathcal{F}_{1,2}^{c r i}= \pm \mathcal{A}_{z}-a(1-z)-b z
$$

where plus and minus signs apply to $\mathcal{F}_{1}^{\text {cri }}$ and $\mathcal{F}_{2}^{\text {cri }}$, respectively. The evolution of $z$ is then governed by consistency conditions. The dynamic equation of the non-linear mass-spring oscillator on which a harmonic forcing amplitude $E_{\max }$ and frequency $\omega$ is exerted has the following expression

$$
\mathcal{M} \ddot{X}+\mathcal{C} \dot{X}+\mathcal{K}(z)\left(X-z \cdot X_{\text {ori }}\right)=E_{\max } \cos (\omega t)
$$

where $\mathcal{M}$ and $\mathcal{C}$ are the mass and damping coefficients, respectively. Temperature evolution is now addressed considering that a spatially homogeneous heat flow exists between the SMA device and the outer domain at temperature $T_{e x}$. Consequently, in the $1 \mathrm{D}$ equivalent form of equation (25), the term involving the divergence of the temperature cancels, and the temperature in the material is characterized by its time evolution only. This choice of modelization is motivated by the fact that the final oscillator model is zero-dimensional; thus, spatial dependence of temperature is disregarded. 
The heat exchange is modelled by a natural convection with a heat exchange coefficient $H$, so that the temperature of the SMA device is now governed by the following equation

$$
\rho C_{P} \dot{T}-A_{z} \dot{z}-\xi T \dot{z}=H\left(T_{e x}-T\right)
$$

In this last equation, the intrinsic dissipation has a simpler form than in the general 3D case because the detwinning process is expected to be fully achieved every time and $|\dot{X}|_{o r i}=0$.

The following non-dimensional parameters are now introduced

$$
\begin{gathered}
\Omega=\frac{\omega}{\omega_{n}}, \quad \tau=\omega_{n} t \\
x=\frac{X}{X_{m s}}, \quad \gamma=\frac{E_{\text {max }}}{F_{m s}} \\
\theta=\frac{T}{T_{r e f}}, \quad \theta_{e x}=\frac{T_{e x}}{T_{r e f}} \\
h=\frac{H}{\rho C_{P} \omega_{n}}, \quad \zeta=\frac{\mathcal{C}}{2 \omega_{n} \mathcal{M}}
\end{gathered}
$$

In these expressions, $\omega_{n} \triangleq \sqrt{\mathcal{K}_{a} / \mathcal{M}}$ is the natural frequency of the oscillator in the austenitic phase, $F_{m s}$ is the force at the beginning of the martensitic phase transformation and $T_{r e f}$ is a reference temperature, in the austenitic phase. Finally, it is assumed that $\mathcal{K}_{a}=\mathcal{K}_{m}$ for simplicity. Finally, the dynamics of the reduced model for the SMA oscillator is governed by the following system

$$
\begin{gathered}
\left.\ddot{x}+2 \zeta \dot{x}+f\left(x, z, x_{\text {ori }}\right)\right)=\gamma \cos \Omega \tau \\
\text { where } f\left(x, z, x_{\text {ori }}\right)=x-z x_{\text {ori }} \\
\text { and } x_{\text {ori }}=x_{\text {max }} \operatorname{sgn}(f) \\
\mathcal{F}_{1}^{c r i t} \leqslant 0, \quad \dot{z} \cdot \dot{\mathcal{F}}_{1}^{\text {crit }}=0 \\
\mathcal{F}_{2}^{\text {crit }} \leqslant 0, \quad \dot{z} \cdot \dot{\mathcal{F}}_{2}^{\text {crit }}=0 \quad \text { where } \mathcal{F}_{1}^{\text {crit }} \\
=\mathcal{A}_{z}-a(1-z)-b z \text { and } \mathcal{F}_{2}^{\text {crit }} \\
=-\mathcal{A}_{z}-a(1-z)-b z \\
\dot{\theta}-\left(\frac{\xi}{\rho C_{P}}\right) \theta \dot{z}-\left(\frac{\mathcal{A}_{z}}{\rho T_{\text {ref }} C_{P}}\right) \dot{z}=h\left(\theta_{e x}-\theta\right)
\end{gathered}
$$

\section{Parameters identification}

The reference article we consider for comparison with our results is that of Lacarbonara et al. (2004) where a thermomechanical SMA model is also used. In the nondimensional version of this model, only four material parameters are considered and denoted by $q_{1}, q_{2}, q_{3}$ and $J$. Their dependency with respect to the parameters in this article is

$$
q_{1}=\frac{\sigma_{m f}}{\sigma_{m s}}, q_{2}=\frac{\sigma_{a f}}{\sigma_{a s}}, q_{3}=\frac{\sigma_{a s}}{\sigma_{m s}}, J=1-\frac{M_{m s}}{T_{r e f}}
$$

where $\sigma_{m s}, \sigma_{m f}, \sigma_{a s}, \sigma_{a f}, M_{m s}, M_{m f}, A_{a s}$ and $A_{a f}$ are stress and temperature thresholds. Subscripts $m s$ and $m f$ and $a s$ and $a f$ refer to forward and reverse transformations, respectively. Our model needs dimensional parameters. Some of them can be chosen arbitrarily, the other ones being calculated from the values of $q_{1}$, $q_{2}, q_{3}$ and $J$. The radius of the cylinder under test is chosen equal to $1 \mathrm{~mm}$, so that $S=3.14 \times 10^{-6} \mathrm{~m}^{2}$; its length $(l)$ is chosen equal to $0.1 \mathrm{~m}$ and the reference temperature $\left(T_{r e f}\right)$ is chosen equal to $296 \mathrm{~K}$. Some material properties are also fixed to realistic quantities of real materials, $\sigma_{m s}=8 \times 10^{8} \mathrm{~Pa}, \sigma_{\text {sori }}=8 \times 10^{7} \mathrm{~Pa}$ (detwinning stress start) and $\sigma_{\text {fori }}=165 \times 10^{6} \mathrm{~Pa}$ (detwinning stress finish). Young's modulus and maximum detwinning strain are set to $E_{a}=E_{m}=5 \times 10^{9} \mathrm{~Pa}$ and $\varepsilon_{\max }=0.112$. Once these quantities are known, all other parameters of the model may be calculated using the following relationships

$$
\begin{aligned}
& X_{m s}=\frac{\sigma_{m s} l}{E a}, M_{m s}=(1-J) T_{r e f}, M_{m f}=\left(1-J q_{1}\right) T_{r e f} \\
& A_{m s}=\left(1-J q_{3}\right) T_{r e f}, A_{m f}=\left(1-J q_{3} \cdot q_{2}\right) T_{r e f} \\
& a=\frac{1}{2}\left[\left(\frac{1}{E_{m}}-\frac{1}{E_{a}}\right) \frac{\sigma_{m s}^{2}-\sigma_{a f}^{2}}{2}+\varepsilon_{\max }\left(\sigma_{m s}-\sigma_{a f}\right)\right] \\
& b=\frac{1}{2}\left[\left(\frac{1}{E_{m}}-\frac{1}{E_{a}}\right) \frac{\sigma_{m f}^{2}-\sigma_{a s}^{2}}{2}+\varepsilon_{\max }\left(\sigma_{m f}-\sigma_{a s}\right)\right] \\
& \beta=\frac{\sigma_{\text {fori }}}{\varepsilon_{\max }}, \alpha=\beta-\frac{\sigma_{\text {sori }}}{\sigma_{\text {fori }}} \varepsilon_{\text {ori }} \\
& G=\frac{1}{2}\left[\left(\frac{1}{E_{m}}-\frac{1}{E_{a}}\right) \frac{\sigma_{m f}^{2}-\sigma_{a f}^{2}-\sigma_{m s}^{2}+\sigma_{a s}^{2}}{2}\right. \\
& \left.+\left(\sigma_{m f}+\sigma_{a s}-\sigma_{m s}-\sigma_{a f}\right) \varepsilon_{\max }-(\alpha-\beta) \varepsilon_{\max }^{2}\right] \\
& x_{\max }=\frac{\varepsilon_{\max } l}{X_{m s}}, T_{0}=T_{r e f}, \kappa=a-\frac{\beta \varepsilon_{\max }^{2}}{2} \\
& \left.C\left(T_{0}\right)=\left(\frac{1}{E_{m}}-\frac{1}{E_{a}}\right) \frac{\sigma_{m s}^{2}+\sigma_{a f}^{2}}{2}+\varepsilon_{\max }\left(\sigma_{m s}+\sigma_{a f}\right)\right) \\
& -\beta \varepsilon_{\max }, \xi=\frac{C\left(\theta_{0}\right)-\kappa}{T_{0}-A_{m f}}
\end{aligned}
$$

Comparison is made, for isothermal oscillations, with two cases of Lacarbonara et al.'s parameter values. Next section will provide the numerical method used for the time integration of equations. 


\section{Numerical scheme}

Time integration is numerically achieved using a nonlinear implicit Newmark scheme adapted to the treatment of the criteria functions for the evolution of the volumic fraction $z$. The main idea is to use a classical Newmark scheme for the mechanical part of the dynamic system. Inside the Newton-Raphson loops, iterations are performed on the two unknowns $z$ and $\theta$, so as to achieve convergence of metallurgic and temperature variables. The main steps are here briefly explained with emphasis on the peculiar treatments needed by the present model. The reader is referred to Géradin and Rixen (1994) and Hughes (2000) for more details on the Newmark scheme. Let us assume that at time $\tau_{n}$, the state variables $\left(x_{n}, \dot{x}_{n}\right.$, $\ddot{x}_{n}, z_{n}, x_{\text {ori }, n}$ and $\theta_{n}$ ) are known. The first step is to predict the displacement, velocity and accelerations at time $\tau_{n+1}=\tau_{n}+\Delta \tau$ with the classical formula

$$
\begin{gathered}
x_{n+1}^{*}=x_{n}+\dot{x}_{n} \Delta \tau+\frac{\Delta \tau^{2}}{2}\left(1-2 \beta_{N}\right) \ddot{x}_{n} \\
\dot{x}_{n+1}^{*}=\dot{x}_{n}+\left(1-\gamma_{N}\right) \Delta \tau \ddot{x}_{n} \\
\ddot{x}_{n+1}^{*}=0
\end{gathered}
$$

with $\left(\gamma_{N}, \beta_{N}\right)$ the classical parameters of the Newmark family. The three remaining variables are kept constant so that $z_{n+1}^{*}=z_{n}, x_{o r i, n+1}^{*}=x_{o r i, n}$ and $\theta_{n+1}^{*}=\theta_{n}$. The residue $\mathcal{R}_{n+1}$ is introduced

$$
\begin{aligned}
\mathcal{R}_{n+1}= & \ddot{x}_{n+1}+2 \zeta \dot{x}_{n+1} \\
& +\left(x_{n+1}-z_{n+1} \cdot x_{\text {ori }, n+1}\right)-\gamma \cos \Omega \tau_{n+1}
\end{aligned}
$$

Table 2 gives an overview of the main steps of the numerical integration. In the numerical steps, we focus on the case of forward transformation for the sake of simplicity. In addition, to ensure Kuhn-Tucker conditions at the discrete level, simultaneous test of the positivity of $\mathcal{F}_{1}^{\text {cri }}$ at time $n: \mathcal{F}_{1}^{\text {cri,n }}>0$ and of $\dot{\mathcal{F}}_{1}^{\text {cri }}$ via $\mathcal{F}_{1}^{c r i, n+1}>\mathcal{F}_{1}^{c r i, n}$ is computed. Enforcing $1-z_{n+1}^{*}>0$, an update of $z_{n+1}^{*}$ is obtained by expanding the criteria function at time $\tau_{n+1}$ at first order $\mathcal{F}_{1}^{c r i, n+1}=\mathcal{F}_{1}^{c r i, n}+\left(\partial \mathcal{F}_{1}^{c r i} / \partial z\right)_{n} \cdot \delta z_{n+1}$.

As cancellation of $\mathcal{F}_{1}^{c r i, n}+{ }^{n}$ is searched, one gets the increment $\delta z_{n+1}$ for correcting the first prediction. In the case of the reverse transformation, one has to substitute $\mathcal{F}_{2}^{c r i}$ for $\mathcal{F}_{1}^{c r i}$ and to enforce $z_{n+1}^{*}>0$ in the previous reasoning. In the numerical calculations, the Newmark parameters have been set to their classical values $\gamma_{N}=1 / 2$ and $\beta_{N}=1 / 4$, while a tolerance $\varepsilon_{e q}$ of $10^{-10}$ has been used. Results are shown in the next section for isothermal and non-isothermal oscillations.

\section{Isothermal oscillations}

Case I. In this section, the numerical results provided by our model are compared to those presented by
Table 2. Numerical scheme for time integration.

I. The convergence criterion is set to $\left|\mathcal{R}_{n}+1\right| \leqslant \varepsilon_{\text {eq }}\left|\mathcal{R}_{n}\right|$

2. Updating of mechanical variables is performed after determining the displacement increment $\left(\left(\frac{\partial \mathcal{R}}{\partial \delta x}\right)_{n} \delta x_{n+1}=-\mathcal{R}_{n}\right.$, where $\left.\left(\frac{\partial \mathcal{R}}{\partial \delta x}\right)_{n}=1+\frac{1}{\beta \Delta \tau^{2}}+2 \zeta \frac{\gamma}{\beta \Delta \tau}\right)$, using Géradin and Rixen (1994)

3. Computation and test of criteria functions corresponding to equation (37)

4. Computation of the increment $\delta z_{n}+1$

5. Updating value of $z_{n+1}=z_{n+1}^{*}+\delta z_{n+1}$

6. Computation of the thermodynamic force $\mathcal{A}_{z, n+1}$

7. Temperature can be predicted from the heat equation discretized by an Euler implicit scheme:

$$
\theta_{n+1}=\frac{\theta_{n+1}^{*}+h \theta_{\text {ex }} d \tau+\left(\frac{\mathcal{A}_{z, n}+1}{\rho T_{\text {ref }} C_{p}}\right)\left(z_{n+1}-z_{n}\right)}{1+h d \tau-\left(\frac{\xi}{\rho C_{p}}\right)\left(z_{n+1}-z_{n}\right)}
$$

8. Computation of the latent heat density $C\left(\theta_{n+1}\right)$ and criteria functions $\mathcal{F}_{\mathrm{I}, 2}^{c r, n+1}$

9. Repeat the process until mechanical equilibrium and criteria are fulfilled

Lacarbonara et al. (2004). In this first case, the following values are considered for the material parameters: $q_{1}=1.3, \quad q_{2}=0.667, \quad q_{3}=0.9 \quad$ and $\quad J=0.315$. Following the identification procedure explained before, the ZM parameters are deduced and given in Table 3. Comparison of results are reported on FRFs, in order to get the most complete picture of the dynamics, in the vicinity of the normalized eigenfrequency. A simple and direct method for obtaining FRFs is used: for each value of the forcing frequency $\Omega \in[0,2]$, a time simulation is performed. Neglecting the transient phase, the maximum amplitude of absolute value of the displacement $|x|_{\max }$ is recorded. For the simulations, the time awaited for the transient to die away has been set to 80 periods of the forcing, while

Table 3. ZM parameters for case $I$, corresponding to the following parameters in Lacarbonara et al. (2004): $q_{1}=1.3$, $q_{3}=0.9, q_{2}=0.667$ and $\mathrm{J}=0.315$.

\begin{tabular}{ll}
\hline Symbol & Value \\
\hline $\mathrm{a}$ & $17.920 \times 10^{6} \mathrm{~Pa}$ \\
$\mathrm{~b}$ & $17.920 \times 10^{6} \mathrm{~Pa}$ \\
$\beta$ & $1.4732 \times 10^{9} \mathrm{~Pa}$ \\
$\alpha$ & $1.4732 \times 10^{9} \mathrm{~Pa}$ \\
$\mathrm{G}$ & $26.88 \times 10^{6} \mathrm{~Pa}$ \\
$Y$ & $164 \times 10^{6} \mathrm{~Pa}$ \\
$\kappa$ & $8.68 \times 10^{6} \mathrm{~Pa}$ \\
$\xi$ & $0.53114 \times 10^{6} \mathrm{~Pa} /{ }^{\circ} \mathrm{C}$ \\
$\varepsilon_{\max }$ & 0.112 \\
$A_{f}$ & $38.5945 \mathrm{~K}$ \\
$E_{a}$ & $5 \times 10^{10} \mathrm{~Pa}$ \\
$E_{m}$ & $5 \times 10^{10} \mathrm{~Pa}$ \\
$\theta_{0}$ & $233.3498 \mathrm{~K}$ \\
\hline
\end{tabular}

ZM: Zaki-Moumni. 


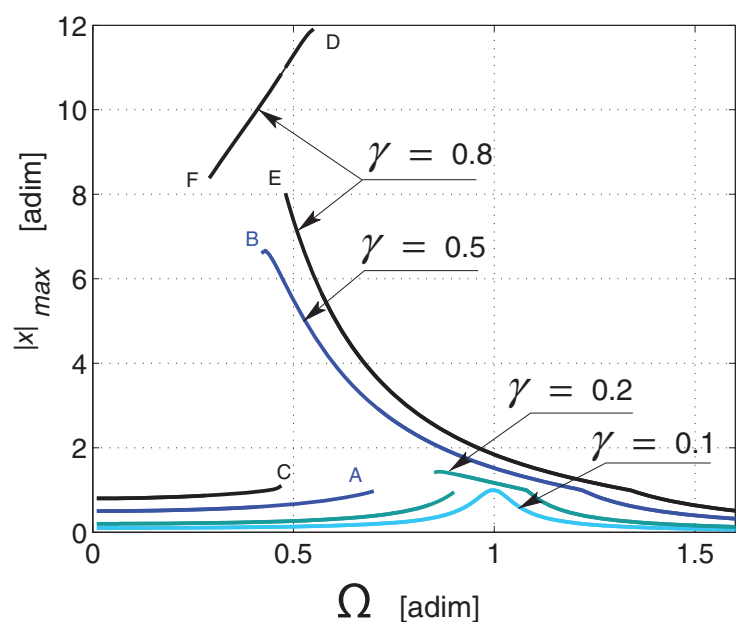

(a)

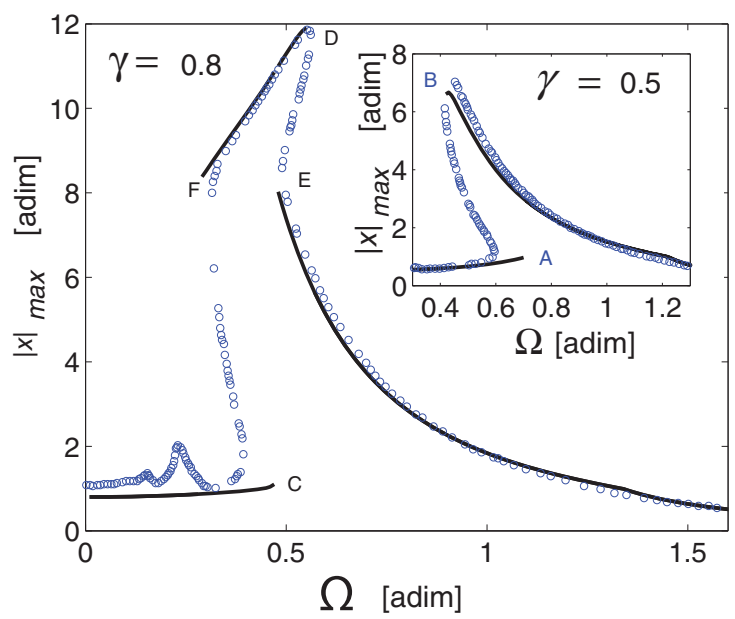

(b)

Figure 7. (a) Maximum amplitude of displacement $|x|_{\max }$ of the device for varying excitation frequencies $\Omega$ and for four amplitudes of forcing: $\gamma=0.1,0.2,0.5$ and 0.8 . (b) Comparison of the results provided by our model (solid line) and those by Lacarbonara et al. (circles), for $\gamma=0.8$ and $\gamma=0.5$ (inset).

adim: adimensional.

the settle time used to pick out the maximum amplitude is 120 periods. Time integration is performed with the adapted Newmark scheme described in section 'Numerical scheme', where the time step is selected as $\delta \tau=2 \pi / 1000 \Omega$, corresponding to 1000 points per periods. In order to get the complete branches of solutions in the non-linear range, the procedure is repeated for increasing and decreasing frequencies. For each forcing frequency $\Omega$, the initial condition selected for the time simulation is taken as the end point of the previous run, in order to minimize the transient time. Figure 7(a) shows the FRFs obtained for four increasing values of the forcing amplitude: $\gamma=0.1,0.2,0.5$ and 0.8 . For $\gamma=0.1$, the amplitude of the response never exceeds 1 , so that no phase transformation occurs: a linear response is observed. For $\gamma=0.2$, non-linear response of the material is excited. As the effective stiffness of the material severely decreases when martensitic transformation begins, a softening behaviour is observed with appearance of jump phenomena, which are enhanced for $\gamma=0.5$. In that case, a first jump is observed at point A when increasing excitation frequency from 0 and the amplitude of the response suddenly increases as the response jumps to the higher branch. On the other hand, when decreasing the excitation frequency and following the higher branch, a jump is observed at point B where the response suddenly goes to very small values. For $\gamma=0.8$, levels of response amplitudes are attained such that the martensitic transformation is completed. This results in the appearance of a third branch of solutions. When increasing $\Omega$ from 0 , a first jump is observed at point $C$ where the solution goes to the third branch, which is left at point $\mathrm{D}$ where a second jump occurs. Decreasing $\Omega$ from 2 , jumps are now observed at points $\mathrm{E}$ and $\mathrm{F}$. The FRFs obtained with our model are completely in the line of previous results reported elsewhere, where the softening-type behaviour and the appearance of the third branch with a hardening-type non-linearity have already been observed by Lacarbonara et al. (2004). A quantitative comparison with their results is shown in Figure 7(b), for $\gamma=0.5$ and 0.8 . They used a refined continuation method so that stable and unstable responses are obtained. This is in contrast with our result where the direct integration only leads to the stable solutions. The quantitative comparison for $\gamma=0.5$ shows a very good agreement, the only noticeable difference being the saddle-node bifurcation at point A occurring later in our model, for $\Omega=0.7$ instead of 0.6 in their work. The second noticeable difference is the maximum amplitude of the main branch, near point $\mathrm{B}$, which is a little bit less in our model. However, all the other features are qualitatively and quantitatively found. The same comparison gives satisfactory results for $\gamma=0.8$, where the saddle-node bifurcation points and the amplitudes of the solution responses are the same. However, a main difference is found in the lowfrequency range, where the model of Lacarbonara et al. predicts occurrence of $1 / 3$ and $1 / 5$ superharmonic resonance whereas our model do not. In our simulations, the amplitude of the response never exceeds 1 for $\Omega$ from 0 to point $\mathrm{C}$, hence no phase transformation occurs and no superharmonic resonance can be excited.

The response is further investigated by increasing the amplitude of the forcing to $\gamma=1.2$. Figure 8(a) shows that the hardening-type behaviour now dominates the response. Superharmonic responses are also now excited in the low-frequency range, resulting in a disordered cloud of points. To get insight into the response in that frequency range, Poincaré section (stroboscopy 


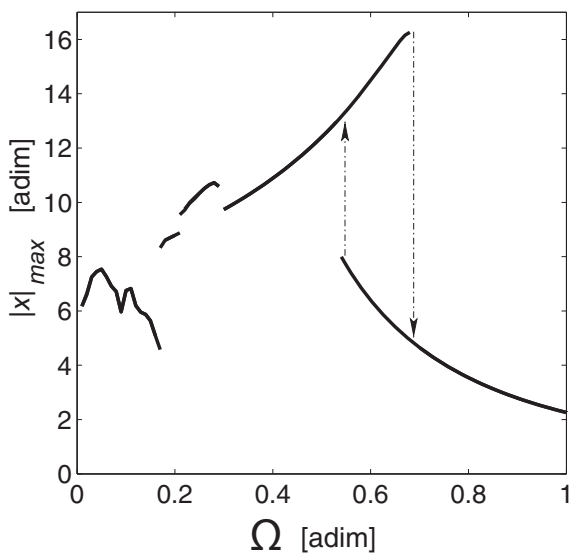

(a)

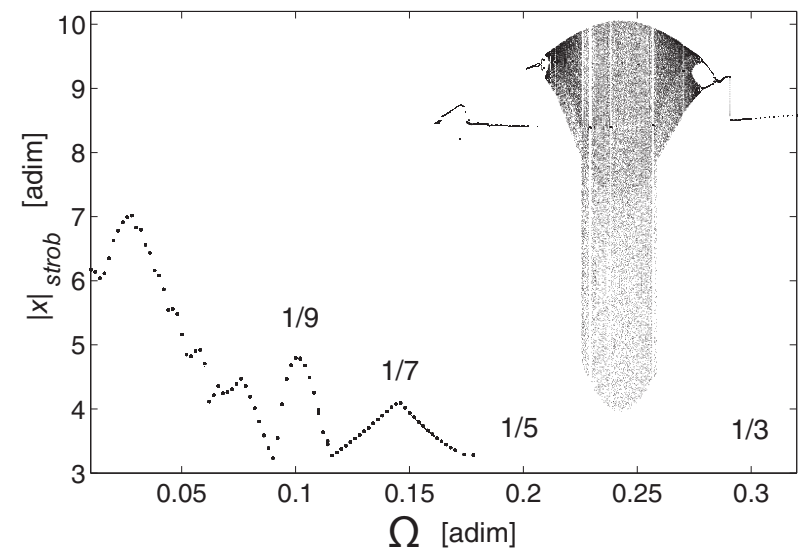

(b)

Figure 8. (a) Maximum amplitude of displacement $|x|_{\max }$ of the device for varying excitation frequencies $\Omega$ and for $\gamma=1.2$ (b) Poincaré section for $\Omega \in[0,0.35]$, showing the appearance of chaotic solutions.

adim: adimensional.

at the forcing frequency) is computed to distinguish periodic from chaotic response, the result is shown in Figure 8(b). The term 'chaos' is used here in its classical definition from dynamical systems theory that can be found from many textbooks (e.g. Guckenheimer and Holmes, 1983; Manneville, 1990; Schuster and Just, 2005), that is, a non-periodic permanent state, characterized by a strange attractor displaying fractal dimension and (at least) one positive Lyapunov exponent. Increasing the excitation frequency from 0 , one can observe clearly the successive appearance of $1 / 9$ and $1 / 7$ superharmonic resonance. Below $\Omega=0.2$, periodic response persists, as indicated by the single point given by the Poincaré section. In the region where $1 / 5$ and $1 / 3$ superharmonic resonance should be excited, a chaotic region is found. At the limiting values of the chaotic region, pitchfork symmetry-breaking bifurcations are observed and followed by period-doubling scenario. The symmetry-breaking bifurcation gives rise to unsymmetrical responses with appearance of even harmonics in the response, whereas the internal force is symmetric. This feature has been first observed in the Duffing oscillator by Parlitz and Lauterborn (1985) and is also reported in the work by Lacarbonara et al. (2004) but for other parameter values, as they did not test such high values of forcing amplitude. The chaotic response is illustrated in Figure 9 for $\Omega=0.23$ and $\gamma=1.2$. The chaotic behaviour is here assessed by the continuous component in the Fourier spectrum (Figure 7(b)) as well as in the self-similar (fractal) appearance of the phase portrait (Figure 7(c)). The period-doubling route observed in Figure 9(b) is also a clear indicator stating the presence of chaotic oscillators at the end of the Feigenbaum scenario (Guckenheimer and Holmes, 1983; Schuster and Just, 2005). Note that a complete characterization of the chaotic nature of the dynamics, which would be ascertained by the presence of (at least) one positive Lyapunov exponent, was not the primary goal of that article. The interested reader can find such developments in Machado et al. (2009) as well as in Lacarbonara et al. (2004) and Sitnikova et al. (2008, 2010). The symmetry-breaking bifurcation is clearly evidenced by the appearance of even harmonics in the response, as shown in Figure 9(b). The chaotic motion is characterized by a chaotic amplitude modulation, whereas a strong persistency of the excitation frequency is observed. The phase portrait underlines the fractal nature of the attractor as well as its asymmetry between positive and negative values of the displacements, resulting from the symmetry-breaking bifurcation. Finally in the restoring force, one can observe an accumulation of straight lines near $x_{M f}$, highlighting the fact that chaotic responses are observed for oscillation amplitudes in the vicinity of the completed martensitic phase.

Case 2. In this section, our model is further confronted to the results presented in Lacarbonara et al. (2004), where an hysteresis loop having nearly flat pseudoelastic plateaus and a larger area is considered by setting, for their model: $q_{1}=1.05, q_{3}=0.3, q_{2}=0.833$ and $J=0.315$. The corresponding ZM parameters are then fixed to the values given in Table 4. Figure 10 shows the frequency-response obtained in that case, for four increasing values of the forcing amplitude, $\gamma=0.1$, $0.2,0.5$ and 0.8 . Results have been quantitatively compared to theirs and show once again very good agreement. In comparison to case 1, all the essential features are found back. The larger area of the hysteresis loop in case 2 with comparison to case 1 induces that more dissipation is at work in the non-linear responses so that the peak amplitudes for each value of $\gamma$ is smaller than those found in case 1. Once again, no 

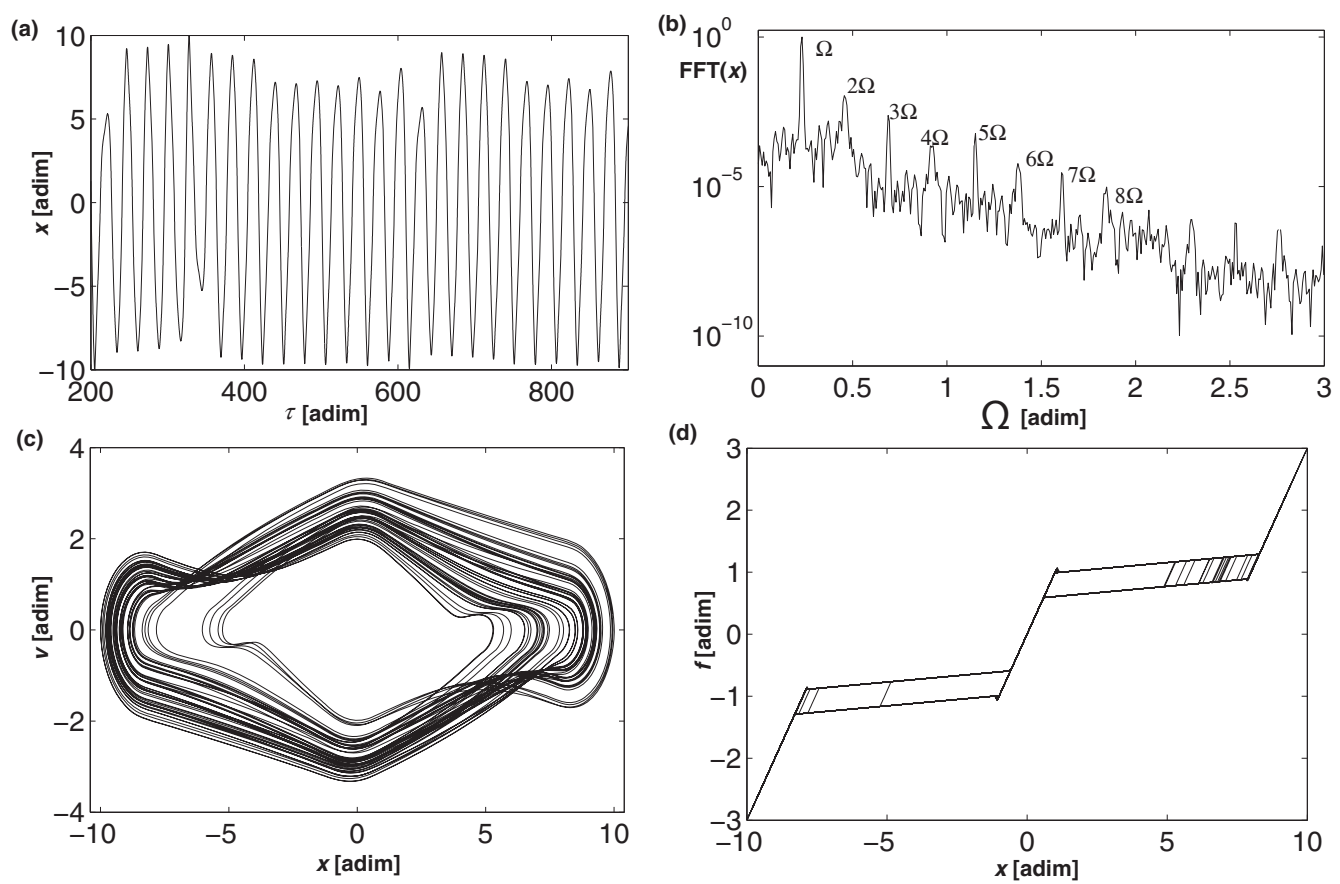

Figure 9. Chaotic response for case I, with $\Omega=0.23$ and $\gamma=1.2$ : (a) time response in the permanent regime, (b) Fourier transform of displacement $x$, (c) phase portrait displacement $x$ versus velocity $v=\dot{x}$ and (d) restoring force of the device as a function of the displacement.

adim: adimensional; FFT: Fast Fourier Transform

Table 4. ZM parameters for case 2, corresponding to the following parameters in Lacarbonara et al., (2004): $q_{1}=1.05$, $q_{3}=0.3, q_{2}=0.833$ and $\mathrm{J}=0.315$.

\begin{tabular}{ll}
\hline Symbol & Value \\
\hline $\mathrm{a}$ & $33.60 \times 10^{6} \mathrm{~Pa}$ \\
$\mathrm{~b}$ & $33.60 \times 10^{6} \mathrm{~Pa}$ \\
$\beta$ & $0.71429 \times 10^{9} \mathrm{~Pa}$ \\
$\alpha$ & $0.71429 \times 10^{9} \mathrm{~Pa}$ \\
$A_{f}$ & $306.7763 \mathrm{~K} \mathrm{~K}$ \\
$Y$ & $16 \times 10^{7} \mathrm{~Pa}$ \\
$\kappa$ & $29.120 \times 10^{6} \mathrm{~Pa}$ \\
$\theta_{0}$ & $264.8183 \mathrm{~K}$ \\
$\mathrm{G}$ & $4.4800 \times 10^{6} \mathrm{~Pa}$ \\
\hline
\end{tabular}

ZM: Zaki-Moumni.

$\varepsilon_{\max }, E_{a}$ and $E_{m}$ have not been reported in the table as they do not change with respect to case I.

superharmonic responses are found below the normalized eigenfrequency for $\gamma=0.8$, which is also the result shown in Lacarbonara et al. (2004). The appearance of a third branch corresponding to completed martensitic transformation is also depicted as in case 1 . Interestingly, this branch splits into two parts, which is in line with the results they presented where chaotic response were exhibited on the low-frequency part of this third, upper branch. A Poincaré section is realized for the corresponding frequency and amplitude range and is shown in the inset of Figure 10, clearly evidencing the fact that chaotic responses are also predicted by our model. The chaotic response is shown in Figure

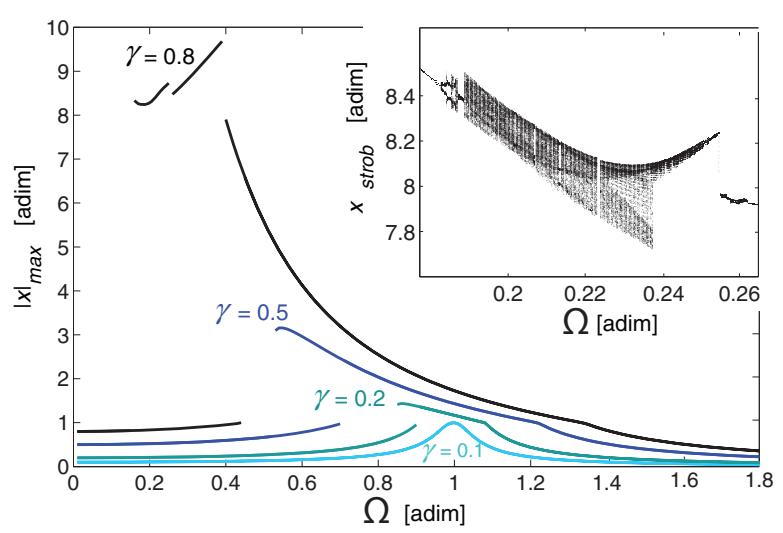

Figure 10. Maximum amplitude of displacement $|x|_{\max }$ for case 2 and for increasing values of the forcing, $\gamma=0.1,0.2,0.5$ and 0.8 . Inset: Poincaré section for $\Omega \in[0.18,0.26]$ and $\gamma=0.8$, corresponding to the solutions found on the upper (third) branch.

adim: adimensional.

11 for $\Omega=0.22$. Once again, the chaotic character of the response is completely enclosed in the temporal variations of the envelope, see Figure 11(a). The time series of the volumic fraction as well as the behaviour of the internal force, shown in Figure 11(d) and (e), respectively, highlights the fact that during the oscillations, the system either fully reached the completed transformation, so that $z$ saturates to 1 , or missed the complete transformation so that $z$ starts to decrease just before reaching 1 . These results show that chaotic 

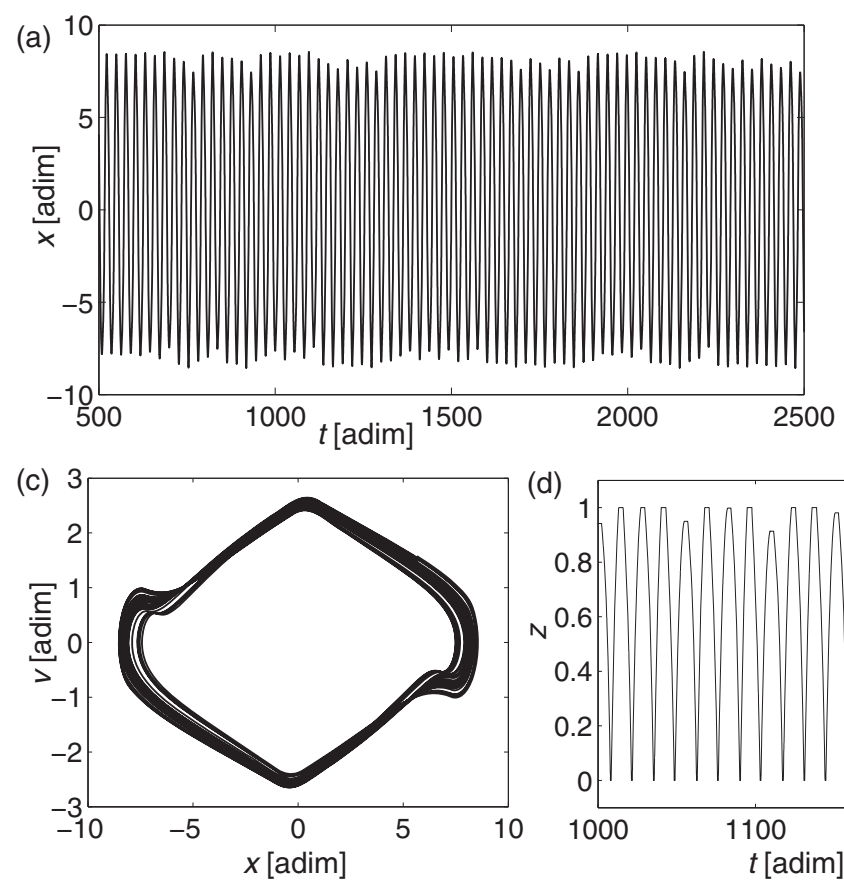
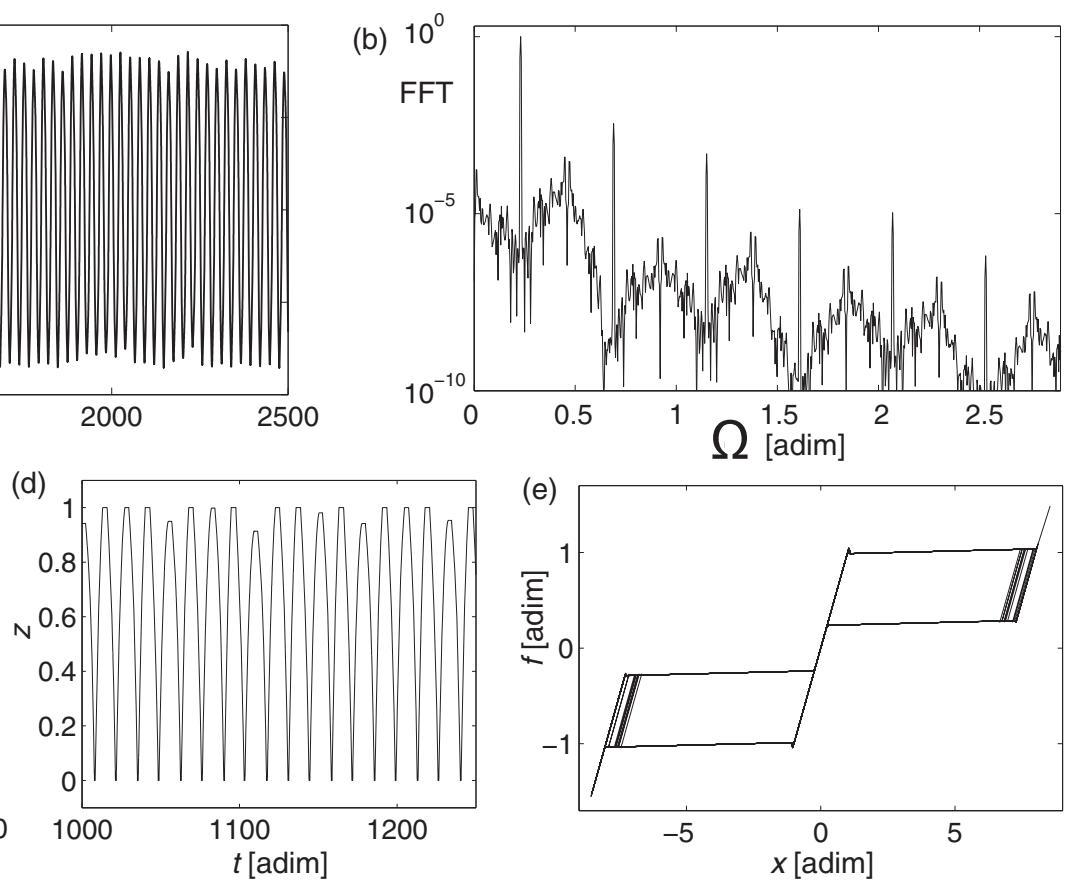

Figure I I. Chaotic response of the device for case $2, \Omega=0.22$ and $\gamma=0.8$. (a) Time series of the displacement $x$ in the permanent regime, (b) FFT of $x$, (c) phase portrait, (d) time series of the volumic fraction $z$ and (e) internal force versus displacement in the permanent regime.

adim: adimensional; FFT: Fast Fourier Transform

responses are generally observed at the limit point where martensitic transformation is completed. Phase portrait and Fourier transformation can be compared to the ones in Lacarbonara et al., showing an impressive agreement between the predictions given by the two models.

Asymmetric restoring force. In this paragraph, the dynamic analysis is carried out in the case of asymmetric constitutive law. Indeed, according to experiments, SMAs clearly exhibit an asymmetric behaviour in tension and in compression (Lexcellent and Rejzner, 2000; Orgéas and Favier, 1998). The ZM model can take this asymmetry into account by introducing the third invariant in the expression of the equivalent detwinning strain (Morin et al., 2011; Zaki, 2010). This asymmetric 3D behaviour law can be reduced to a $1 \mathrm{D}$ law following the procedure explained in section 'Reduced model for a uniaxial SMA oscillator'. After reduction, the parameters defining the asymmetric behaviour are now given by two values for $\varepsilon_{\max }$, one for tensile loading $\varepsilon_{\max }^{t}$ and one for compression $\varepsilon_{\max }^{c}$. In this section, the model parameters of case 1 have been selected, except the values of $\varepsilon_{\max }$ that have been set to $\varepsilon_{\max }^{t}=0.112$ and $\varepsilon_{\max }^{c}=0.064$, respectively. As compared to the symmetric case studied in previous sections, the compression loop has been shortened and the plateaus made more stiff. The behaviour of this asymmetric device is shown in Figure 12(b). The frequencyresponse curve for an excitation amplitude of $\gamma=0.5$ is shown in Figure 12(a) where the maximum amplitude is compared to the symmetric case. As the behaviour has been selected more stiff in compression, the softening behaviour is less important than in the symmetric case. Asymmetry is also observable by the fact that maximum and minimum values of the displacement are now different. Inset in Figure 12(a) shows minus the minimum displacement (-xmin) for comparison. Figure 12(c) shows the time series of the displacement for $\Omega=0.6$, where this asymmetry is also clearly evidenced: one can see different values of maximum displacement in tensile loading and compression, respectively. Consequently, the Fourier spectrum of the displacement now shows odd and even harmonics, whereas the displacement corresponding to symmetric restoring force contains only odd harmonics, as shown in Figure 12(d). This shows that the model is able to reproduce the more realistic case of an asymmetric restoring force and reproduce the expected features corresponding to that case.

\section{Non-isothermal oscillations}

As shown previously, SMAs display thermomechanical coupling, which is responsible for numerous important effects that have already been underlined (Bernardini and Rega, 2009; Bernardini and Vestroni, 2003; He and Sun, 2011; Morin et al., 2011). Hence, it is necessary to take into account this phenomena, when dealing with the dynamic behaviour of the material. The 

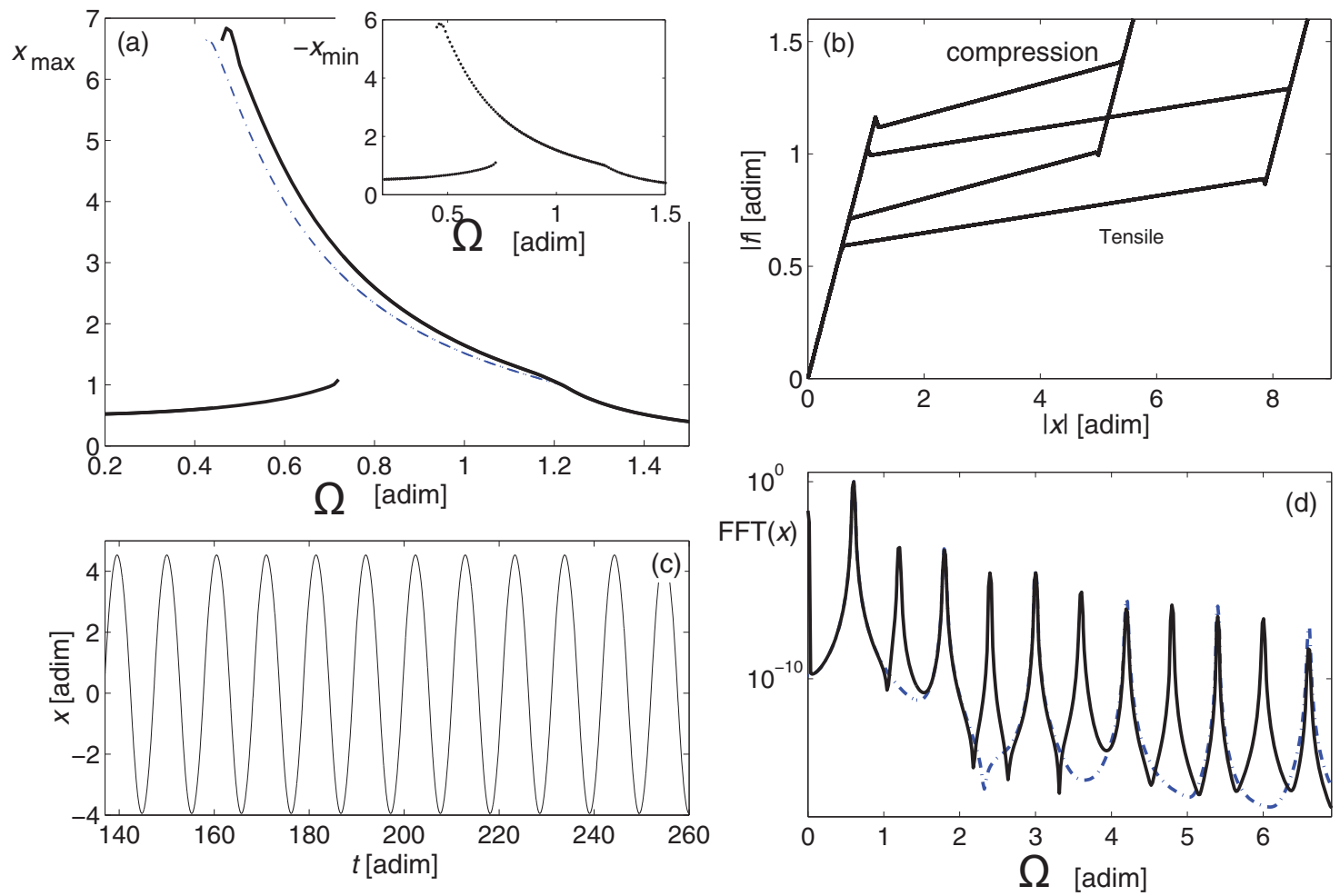

Figure I2. Asymmetric behaviour of the pseudoelastic device. (a) Frequency-response curve $x_{\max }$ (inset: $x_{\min }$ ) versus excitation frequency $\Omega$, for $\gamma=0.5$, (b) asymmetric behaviour (solid line) is compared to the corresponding symmetric behaviour (dash-dotted line), (c) time response $x$ in the permanent regime for $\Omega=0.6$, upper branch and (d) Fourier transform of the displacement $x$ (solid line), compared to the symmetric case (dash-dotted line).

adim: adimensional; FFT: Fast Fourier Transform

equations of motions now include the temperature variations as shown in section 'Reduced model for a uniaxial SMA oscillator'. Heat sources are the sum of a term proportional to $\dot{z}$, describing heat release and absorption due to the martensitic forward and reverse transformations (latent heat), together with a term proportional to $h$, and describing the heat exchange with external environment. It is worth noting that the current contribution neglects thermoelastic coupling effect. Moreover, choosing the value of the material convective coefficient $h$ is inspected in Figure 13, showing the forced response of the non-isothermal system for a forcing amplitude of $\gamma=0.8$ and a frequency $\Omega=0.8$. Material parameters, unless specified, are those from case 1 of the previous section (see Table 5 for additional parameters). In particular, a symmetric internal restoring force is selected. For $h=0$, an adiabatic system is at hand. As no thermal energy is transferred to the environment, the heat generated by the martensitic transformation remains in the device that continuously warms up. Hence, no stable state solutions exist for the temperature, so that this non-realistic case is not prone to a study on permanent states. As soon as $h>0$, energy exchange with environment makes possible the existence of a permanent regime where the temperature stabilizes at a mean constant value. For increasing values of $h$, one can observe that (a) the transient regime is shorter (for $h=0.01$, permanent regime is obtained after time $\tau=1000$ ); (b) the mean temperature stabilizes at a smaller value and (c) amplitude of oscillations around this mean value also decreases. These three observations are completely in the lines of the thermal behaviour and the equilibrium between the device and the external environment, governed by $h$. The frequency of the oscillations in $\theta$ is also seen to be twice that of the displacement $x$, since for one cycle of the device, including tension and compression, two cycles for the temperature are realized. Finally, Figure 13 clearly highlights the fact that the behaviour of $\theta$ is almost completely driven by that of the volumic fraction of martensite $z$.

As highlighted in section 'Model equations', the temperature is prone to show important variations, especially during a simulation for constructing an FRF where the initial condition of a new run is selected as the last values of the precedent (see section 'Isothermal oscillations'). Before showing the numerical frequencyresponse curves obtained in the non-isothermal case, the most important effects of initial temperature and thermomechanical coupling are shown in Figure 14.

Figure 14(a) highlights the fact that for two increasing values of the initial temperature $\left(\theta_{1}^{0}=40^{\circ} \mathrm{C}<\right.$ 

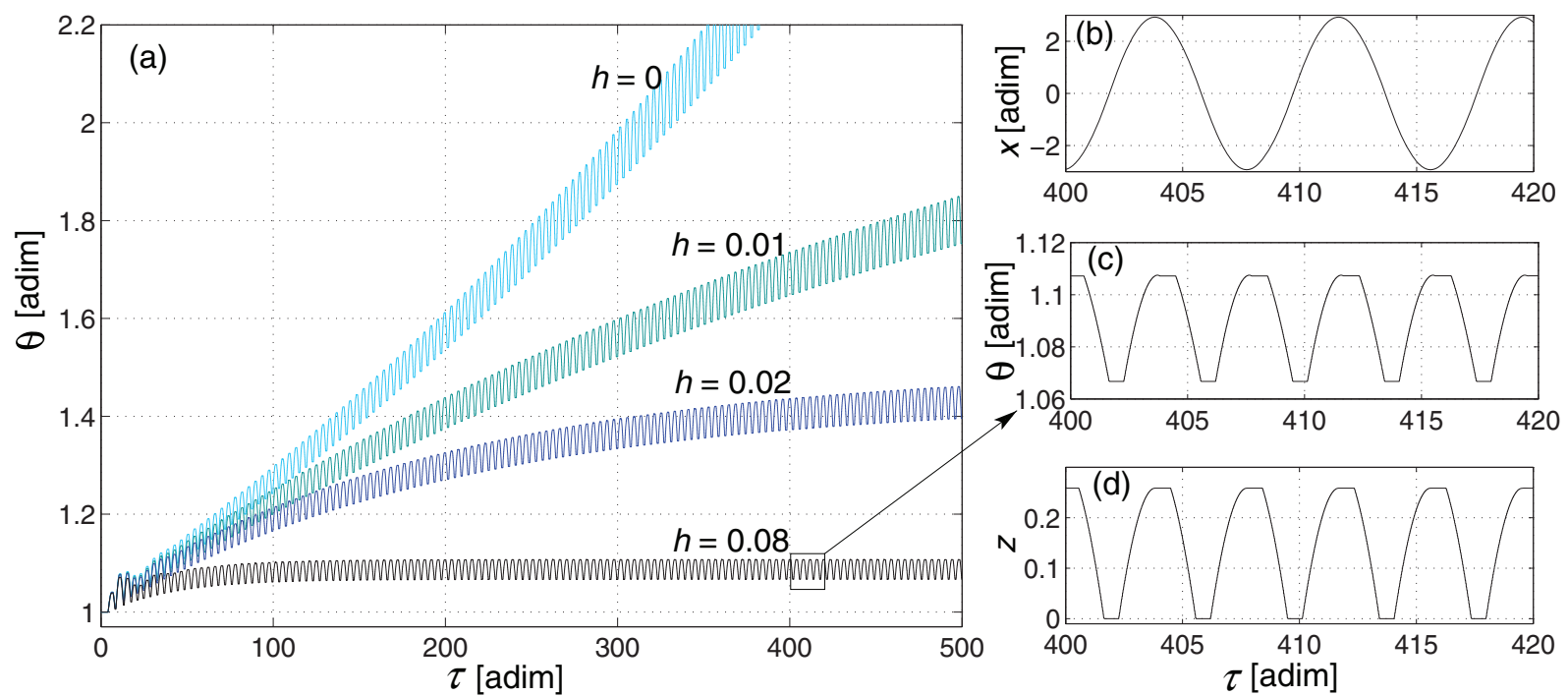

Figure 13. Non-isothermal behaviour of the shape memory device. (a) Time series of the non-dimensional temperature $\theta$ when the device is forced harmonically with an amplitude $\gamma=0.8$ and a frequency $\Omega=0.8$, for four increasing values of $h$ : 0 (adiabatic), $0.01,0.02$, and 0.08; (b) displacement $x$ versus time for $h=0.008$ in the permanent regime; (c) temperature $\theta$ and (d) volumic fraction $z$ at the same instants of time.

adim: adimensional.

Table 5. Additional material parameters (specific heat capacity $C_{P}$, density $\rho$ and external medium temperature $T_{\text {ext }}$ )

\begin{tabular}{ll}
\hline Symbol & Value \\
\hline$C_{P}$ & $440 \mathrm{~J} \mathrm{~K}^{-1} \mathrm{~m}^{-3} \mathrm{~kg}^{-1}$ \\
$\rho$ & $6500 \mathrm{~kg} \mathrm{~m}^{-3}$ \\
$T_{\text {ext }}$ & $333 \mathrm{~K}$ \\
\hline
\end{tabular}

$\left.\theta_{2}^{0}=53^{\circ} \mathrm{C}\right)$, force thresholds and loop area increase. In addition, the already underlined features (thermal hardening and loop area variation) are retrieved in the behaviour of the device, as shown in Figure 14(b) that compares isothermal and non-isothermal stress-strain relationships, for an initial temperature $\theta_{i}=40^{\circ} \mathrm{C}$.

Finally, frequency-response curves are shown in Figure 15 for two different values of $h$. Because of thermomechanical coupling, as illustrated in Figure 13, transients are now longer as compared to the isothermal case. Hence, the number of periods awaited for the transient to die away has been set to 320 , and the settle time for recording the maximum amplitude is set to 80 periods. This results in simulation times significantly longer. As compared to the isothermal case, softening effect is less pronounced for $h=0.008$. For decreasing values of $h$, one can see the disappearance of the third

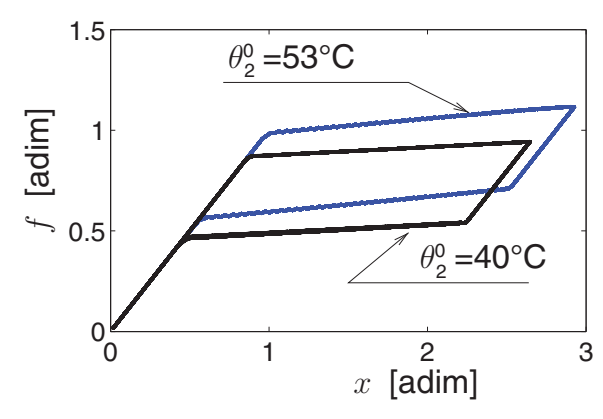

(a)

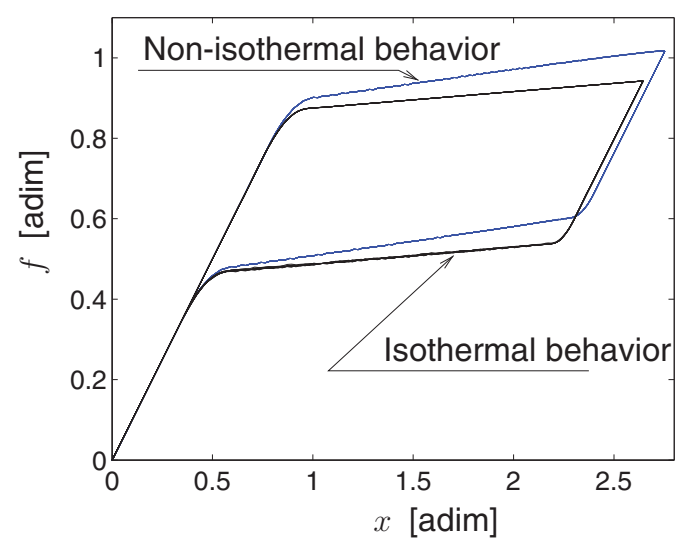

(b)

Figure 14. Non-linear restoring force in non-isothermal conditions (only positive part is shown and negative part is taken as symmetric). (a) Initial temperature effect on mechanical response and (b) comparison between non-isothermal and isothermal behaviours $(h=0.08)$.

adim: adimensional. 


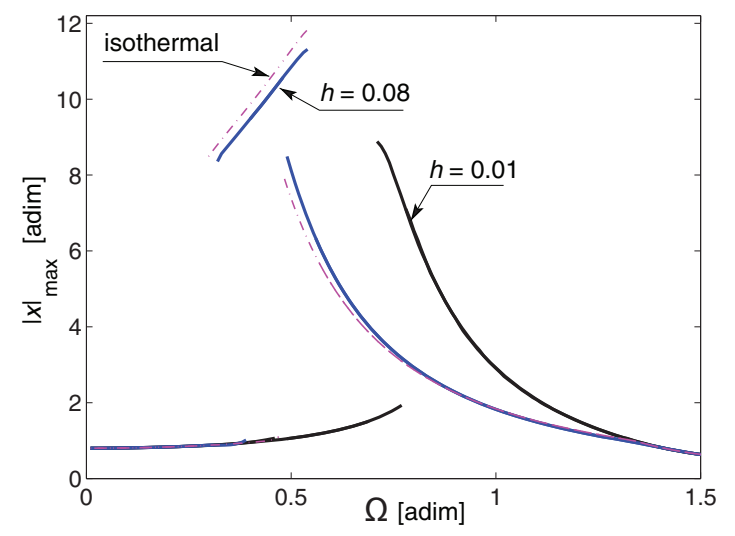

Figure 15. Maximum amplitude of displacement $|x|_{\max }$ of the device for varying excitation frequencies $\Omega$. Isothermal conditions (dash-dotted line) is compared to non-isothermal case with $h=0.008$ and $h=0.0 \mathrm{I}$.

adim: adimensional.

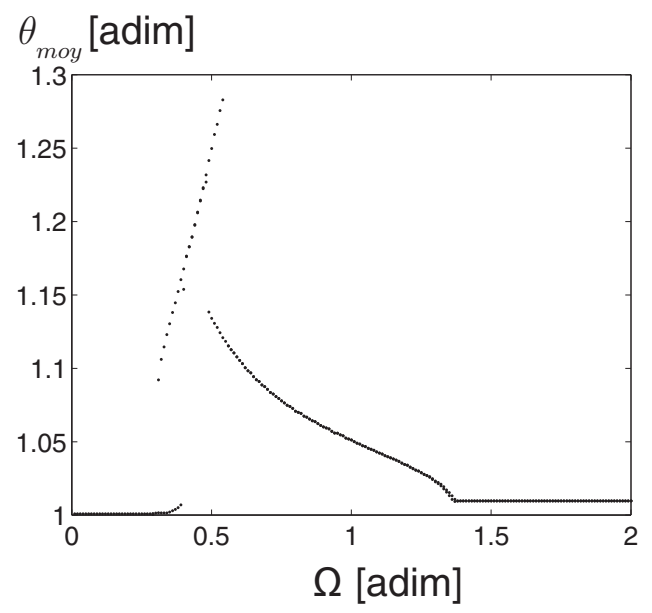

(a) upper branch occurring for $h=0.001$. Small values of $h$ mean that the material does not get enough time to evacuate all heat and lead to an increase of the temperature, and as highlighted above, the phase transformation does not complete.

Figure 16(a) and (b) shows the frequency-response of the mean value $\left(\theta_{\text {moy }}=1 / N\left(\sum_{i=1}^{N} \theta\left(\tau_{i}\right)\right)\right.$, where $N$ is the remainder periods in permanent regime) of the temperature and the temperature variation $(\Delta \theta=$ $\left.\theta_{\max }-\theta_{\text {moy }}\right)$, respectively. One can observe similar features as that of maximum displacement: jump phenomenon, bifurcation at the frequency phase change onset and the emergency of a third stable branch.

\section{Dissipation as a function of loading frequency}

This section investigates the variations of the dissipated energy in the SMA device, with respect to loading

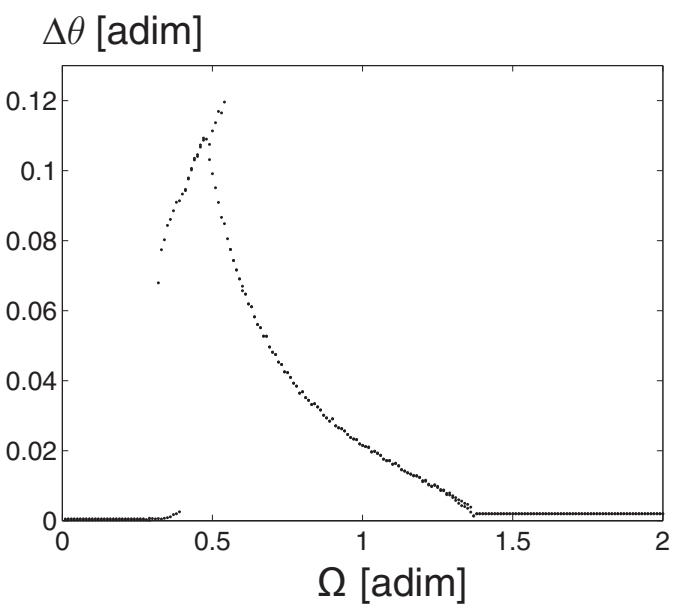

(b)

Figure 16. Frequency-response of material temperature in non-isothermal conditions for $h=0.08$ and $\gamma=0.8$ : (a) mean value $\theta_{\text {moy }}$ and (b) temperature variation $\Delta \theta$.

adim: adimensional.

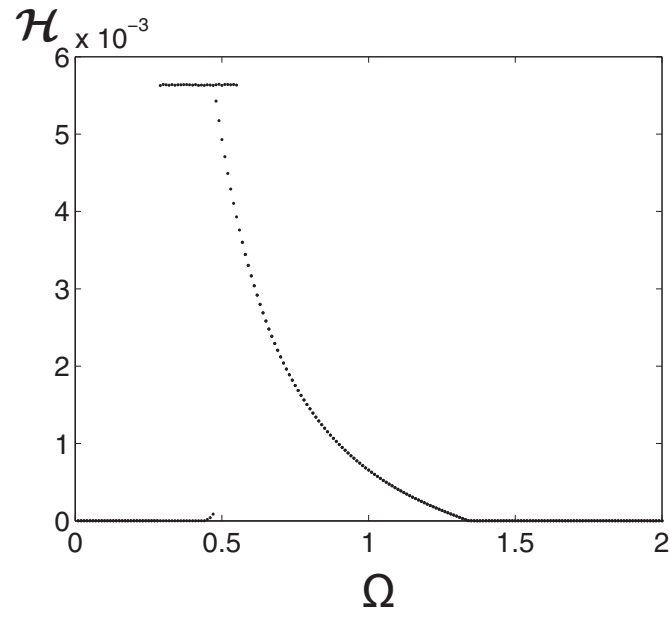

(a)

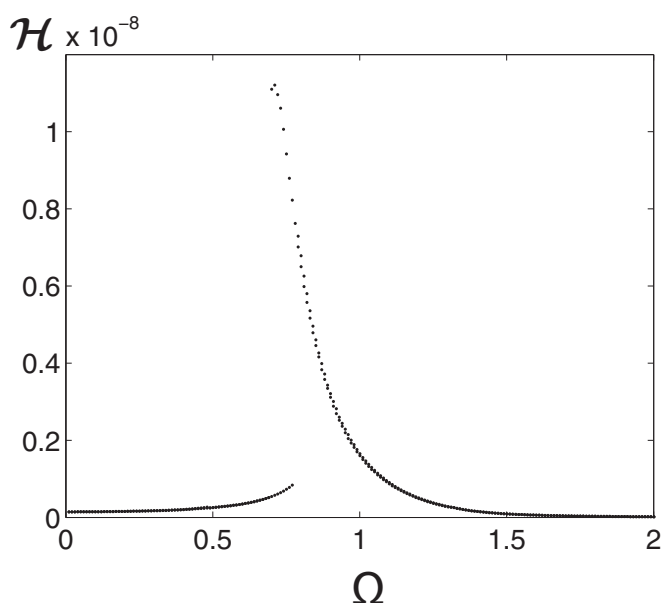

(b)

Figure 17. Area loop $\left(\mathrm{m}^{-3}\right)$ at $\gamma=0.8$ : (a) isothermal case and (b) non-isothermal case $(h=0.0 \mathrm{I})$. 
frequency in both isothermal and non-isothermal cases. The dissipated energy is defined by the area of the hysteresis loop under one cycle $(\oint f \dot{x} d t)$. Figure 17(a) shows a constant value of dissipated energy (area of hysteresis loop) remaining in the frequency range $\left[\begin{array}{ll}0.2 & 0.57\end{array}\right]$. That is due to the accomplishment of phase change and the absence of viscosity. Indeed, the loop area does not change with respect to loading frequency.

In the case of non-isothermal oscillations (Figure 17 (b)), the area of the hysteresis loop does not reach a third stabilized branch in frequency-response and witnesses a decrease as compared to the isothermal case in the same frequency range. One can highlight that for small values of $h$, increasing loading amplitude could allow to reach a stabilized value of the dissipated energy.

\section{Conclusion}

The model was extended to account for thermomechanical coupling as a means for describing strain rate dependency. A reduced version was then used to simulate the response of a single-degree-of-freedom SMA oscillator. The simulations were shown to fit experimental data taken from Lacarbonara et al. (2004). Time integration of the equations of motion using the reduced model was accomplished using a Newmark scheme. Deviation from experimental data was discussed, and new results were obtained for higher forcing and for asymmetric restoring force. Non-linear features of the damper response were observed, including jumps, period-doubling, symmetry-breaking bifurcations and chaotic responses. The influence of thermomechanical coupling on the frequency-response and on dissipation is studied.

The ZM model, as extended in this article, can now be used to simulate the dynamic response of SMA structures. Future work will focus on modelling a torsional pendulum system, like the one in Doaré et al. (2011) and Sbarra et al. (2011).

\section{Acknowledgements}

The authors would like to thank P. Riberty for his help in mechanical experiments and Lahcene Cherfa for designing experiment specimens.

\section{Funding}

This work is funded by the FNR (Fonds National de Recherche) via CRP HENRI TUDOR center at Luxembourg.

\section{References}

Bernardini D (2001) On the macroscopic free energy functions for shape memory alloys. Journal of the Mechanics and Physics of Solids 49: 813-837.
Bernardini D and Pence TJ (2005) Uniaxial modeling of multi-variants shape memory materials with internal sublooping using dissipation functions. Meccanica 40: 339-364.

Bernardini D and Rega G (2009) The influence of model parameters and of the thermomechanical coupling on the behavior of shape memory devices. International Journal of Non-Linear Mechanics 45: 933-946.

Bernardini D and Vestroni F (2003) Non-isothermal oscillations of pseudoelastic devices. International Journal of Non-Linear Mechanics 38: 1297-1313.

Chrysochoos A, Pham H and Maisonneuve O (1996) Energy balance of thermoelastic martensite transformation under stress. Nuclear Engineering 162(1): 1-2.

Collet M, Foltête E and Lexcellent C (2001) Analysis of the behavior of a shape memory alloy beam under dynamical loading. European Journal of Mechanics-A/Solids 20: 615630.

Doaré O, Sbarra A, Touzé C, et al. (2011) Experimental analysis of the quasi-static and dynamic behavior of shape memory alloys. International Journal of Solids and Structures 49: 32-42.

Feng ZC and Li DZ (1996) Dynamics of a mechanical system with a shape memory alloy bar. Journal of Intelligent Material Systems and Structures 7(4): 399-410.

Funakubo H (1987) Shape Memory Alloys. Precision Machinery and Robotics, vol. 1. CRC Press. Gordon and Breach Science Publishers.

Géradin M and Rixen D (1994) Mechanical Vibrations: Theory and Application to Structural Dynamics. Chichester: Wiley.

Grabe C and Bruhns OT (2008) On the viscous and strain rate dependent behavior of polycrystalline NiTi. International Journal of Solids and Structures 45(7-8): 1876-1895.

Guckenheimer J and Holmes P (1983) Nonlinear Oscillations, Dynamical Systems and Bifurcations of Vector Fields. New York: Springer-Verlag.

Hartl DJ, Mooney JT, Lagoudas DC, et al. (2010) Use of a Ni60Ti shape memory alloy for active jet engine chevron application. I. Thermomechanical characterization. Smart Materials and Structures 19(1): 015020 (14 pp).

He YJ and Sun QP (2011) On non-monotonic rate dependence of stress hysteresis of superelastic shape memory alloy bars. International Journal of Solids and Structures 48: $1688-1695$.

Helm O and Haupt P (2002) Thermomechanical representation of the multiaxial behavior of shape memory alloys. In: Lynch CS (ed.) Proceedings of SPIE: Smart Structure and Materials 2002: Active Materials: Behavior and Mechanics, vol. 4699. Society of Photo Optical, pp. 343-354.

Hughes T (2000) The Finite Element Method: Linear Static and Dynamic Finite Element Analysis. Dover Publications.

Humbeeck JV and Delaey L (1981) The influence of strainrate, amplitude and temperature on the hysteresis of a pseudoelastic $\mathrm{Cu}-\mathrm{Zn}-\mathrm{Al}$ single crystal. Journal de Physique IV 42: 1007-1011.

Lacarbonara W, Bernardini D and Vestroni F (2004) Nonlinear thermomechanical oscillations of shape memory devices. International Journal of Solids and Structures 41: 1209-1234.

Lagoudas D, Machado L and Lagoudas M (2005) Nonlinear vibration of a one-degree of freedom shape memory alloy 
oscillator: a numerical-experimental investigation. In: Structural dynamics and materials conference. Austin, TX, 18-22 April.

Leo PH, Shield TW and Bruno OP (1993) Transient heat transfer effects on the pseudoelastic behavior of shape memory wires. Acta Metallurgica and Materialia 41(8): 2477-2485.

Lexcellent C and Rejzner J (2000) Modeling of the strain rate effect, creep and relaxation of a NiTi shape memory alloy under tension (compression)-torsional proportional loading in the pseudoelastic range. Smart Materials and Structures 9: 613-621.

Lin P, Tobushi H, Tanaka K, et al. (1996) Influence of strain rate on deformation properties of TiNi shape memory alloy. JSME International Journal Series A-Mechanics and Material Engineering 39(1): 117-123.

Machado LG, Lagoudas DC and Savi MA (2009) Lyapunov exponents estimation for hysteretic systems. International Journal of Solids and Structures 46: 1269-1286.

Machado LG, Savi MA and Pacheco PMCL (2003) Nonlinear dynamics and chaos in coupled shape memory oscillators. International Journal of Solids and Structures 40(19): 5139-5156.

Manneville P (1990) Dissipative structures and weak turbulence. Boston: Academic Press.

Morin C, Moumni Z and Zaki W (2011) A constitutive model for shape memory alloys accounting for thermomechanical coupling. International Journal of Plasticity 27: 748-767.

Moumni Z (1995) Sur la modélisation du changement de phase à l état solide. $\mathrm{PhD}$ Thesis, École Nationale Supérieure des Ponts et Chaussées, Paris, France.

Moumni Z, Zaki W and Nguyen QS (2008) Theoretical and numerical modeling of solid-solid phase change: application to the description of the thermomechanical behavior of shape memory alloys. International Journal of Plasticity 24: 614-645.

Mukherjee K, Sircar S and Dahotre NB (1985) Thermal effects associated with stress-induced martensitic transformation in Ti-Ni alloy. Materials Science and Engineering 74: 75-84.

Nemat-Nasser S and Wei-Guo G (2006) Superelastic and cyclic response of NiTi SMA at various strain rate and temperatures. Mechanics of Materials 38(5-6): 463-474.

Orgéas L and Favier D (1998) Stress-induced martensitic transformation of a NiTi alloy in isothermal shear, tension and compression. Acta Materialia 46: 5579-5591.

Parlitz U and Lauterborn W (1985) Superstructure in the bifurcation set of the Duffing equation. Physics Letters $A$ 107(8): 351-355.

Patoor E and Berveiller M (1990) Les Alliages à Mémoire de Forme. Paris: Hermes.
Qidwai MA and Lagoudas DC (2000) On thermomechanics and transformation surfaces of polycrystalline shape memory alloy materials. International Journal of Plasticity 16: 1309-1343.

Saadat S, Salichs J, Noori M, et al. (2002) An overview of vibration and seismic applications of NiTi shape memory alloy. Smart Materials and Structures 11(2): 218-229.

Savi MA and Pacheco PMCL (2002) Chaos and hyperchaos in shape memory systems. International Journal of NonLinear Mechanics 12: 645-657.

Savi MA, Pacheco PMCL and Braga AMB (2002) Chaos in a shape memory two-bar truss. International Journal of NonLinear Mechanics 37: 1387-1395.

Savi MA, Sa MAN, Paiva A, et al. (2008) Tensile-compressive asymmetry influence on shape memory alloy system dynamics. Chaos, Solitons and Fractals 36: 828-842.

Sbarra A, Doaré O, Moussa MO, et al. (2011) Dynamical behaviour of a shape memory alloy torsional pendulum. In: Proceedings of ENOC 2011, Rome, Italy, 24-29 July.

Schmidt I and Lammering R (2004) The damping behaviour of superelastic NiTi components. Materials Science and Engineering $A$ 378: 70-75.

Schuster HG and Just W (2005) Deterministic Chaos: An Introduction. WILEY-VCH Verlag GmbH \& Co. KGaA, Weinheim, Fourth revised and augmented edition.

Seelecke S (2002) Modeling the dynamic behavior of shape memory alloys. International Journal of Non-Linear Mechanics 37(8): 1363-1374.

Shaw JA and Kyriakides S (1995) Thermomechanical aspects of NiTi. Journal of the Mechanics and Physics of Solids 43(8): 1243-1281.

Sitnikova E, Pavlovskaia E and Wiercigroch M (2008) Dynamics of an impact oscillator with SMA constraint. European Physical Journal Special Topics 165: 1269-1286.

Sitnikova E, Pavlovskaia E, Wiercigroch M, et al. (2010) Vibration reduction of the impact system by an SMA restraint: numerical studies. International Journal of Nonlinear Mechanics 45: 837-849.

Zaki W (2010) An approach to modeling tensile-compressive asymmetry for martensitic shape memory alloys. Smart Materials and Structures 19: 025009 (7 pp).

Zaki W and Moumni Z (2007a) A 3D model of the cyclic thermomechanical behavior of shape memory alloys. Journal of the Mechanics and Physics of Solids 55(11): 2427-2454.

Zaki W and Moumni Z (2007b) A three-dimensional model of the thermomechanical behavior of shape memory alloys. Journal of the Mechanics and Physics of Solids 55(11): 2455-2490

Zaki W, Zamfir S and Moumni Z (2010) An extension of the ZM model for shape memory alloys accounting for plastic deformation. Mechanics of Materials 42: 266-274. 